Revue des patrimoines

$40 \mid 2019$

Lits historiques. Première anthologie des lits européens du XVe au XIXe siècle

\title{
Le lit de la première chambre à coucher de Louis II de Bavière au château de Linderhof
}

The Bed in Ludwig II's first Bedroom at the Linderhof Palace

\section{Sybe Wartena}

\section{(2) OpenEdition}

Journals

Édition électronique

URL : http://journals.openedition.org/insitu/23965

DOI : 10.4000/insitu.23965

ISSN : 1630-7305

Éditeur

Ministère de la Culture

Référence électronique

Sybe Wartena, «Le lit de la première chambre à coucher de Louis II de Bavière au château de Linderhof », In Situ [En ligne], 40 | 2019, mis en ligne le 19 septembre 2019, consulté le 14 novembre 2019. URL : http://journals.openedition.org/insitu/23965; DOI : 10.4000/insitu.23965

Ce document a été généré automatiquement le 14 novembre 2019.

\section{cc) () $९$}

In Situ Revues des patrimoines est mis à disposition selon les termes de la licence Creative Commons Attribution - Pas d'Utilisation Commerciale - Pas de Modification 4.0 International. 


\section{Le lit de la première chambre à coucher de Louis II de Bavière au château de Linderhof}

The Bed in Ludwig II's first Bedroom at the Linderhof Palace

Sybe Wartena

Cet article a été traduit par Catherine Gros, conservatrice en chef du patrimoine, centre André Chastel.

Le roi Louis II de Bavière (1845-1886, régnant à partir de 1864) est considéré comme l'un des principaux initiateurs de l'épanouissement de l'artisanat d'art dans le dernier tiers du xix ${ }^{e}$ siècle dans le sud de l'Allemagne. Avec les commandes pour ses créations historicistes de Neuschwanstein, Linderhof et Herrenchiemsee et pour d'autres constructions et projets orientalisants et fantastiques, il exigea de ses artistes et artisans des travaux d'une grande qualité d'exécution. Certains d'entre eux firent de Munich un centre de production artistique d'excellence pour des décennies. Cette contribution porte sur la genèse du lit de la première chambre à coucher de Linderhof ${ }^{1}$ et la façon dont elle s'intègre dans l'histoire des projets des châteaux de Linderhof et Herrenchiemsee.

\section{Les projets de constructions « absolutistes » de Louis II et le rôle particulier de la chambre à coucher}

2 C'est en 1868 que Louis II commença à s'intéresser à un projet de construction pour le pavillon de chasse de son père à Linderhof, dans la vallée de Graswang, dans les Alpes d'Allgäu. Ce bâtiment devait citer Versailles, que Louis II avait visité en 1867, d'abord en miniature. Louis II choisit pour nom de code du projet une anagramme de la maxime du roi de France Louis XIV "L'État c'est moi », «Tmeicos Ettal», qui renvoyait aussi au monastère voisin d'Ettal. Le projet prenant de plus en plus d'ampleur, il ne fut plus possible de le réaliser dans la vallée de Graswang et le roi finit par acheter, en 
septembre 1873, Herreninsel (« l'île des Hommes »), sur le lac du Chiemsee, où il y avait suffisamment d'espace disponible; les travaux commencèrent en 1878. Avec de nouvelles modifications de plan, - jusqu'en 1883, on compte 18 changements successifs - on finit par obtenir une copie exacte du château de Versailles.

3 À Linderhof, le roi projetait parallèlement un deuxième édifice, qui fut agrandi plusieurs fois et qui, exactement comme le projet visant à Versailles, devait être le décor de l'image idéale de la monarchie absolue française. Sur un plan du 30 septembre 1870, pour la première fois, on observe une petite extension de trois pièces à la " petite maison du roi ", le pavillon de chasse de Maximilien II. Dès que cette extension composée d'une pièce ovale et de deux petites pièces en fer-à-cheval fut achevée, on planifia une aile correspondante, disposée parallèlement, et une chambre à coucher reliant ces deux ailes au nord. Il en résulta donc une petite construction à trois ailes, distincte de l'ancien pavillon de chasse, qui en constituait la quatrième, au sud (fig. 1). Les trois nouvelles ailes, de style néo-rococo, furent achevées fin 1872, mais dès 1873, la chambre à coucher fut agrandie et modifiée.

Figure 1

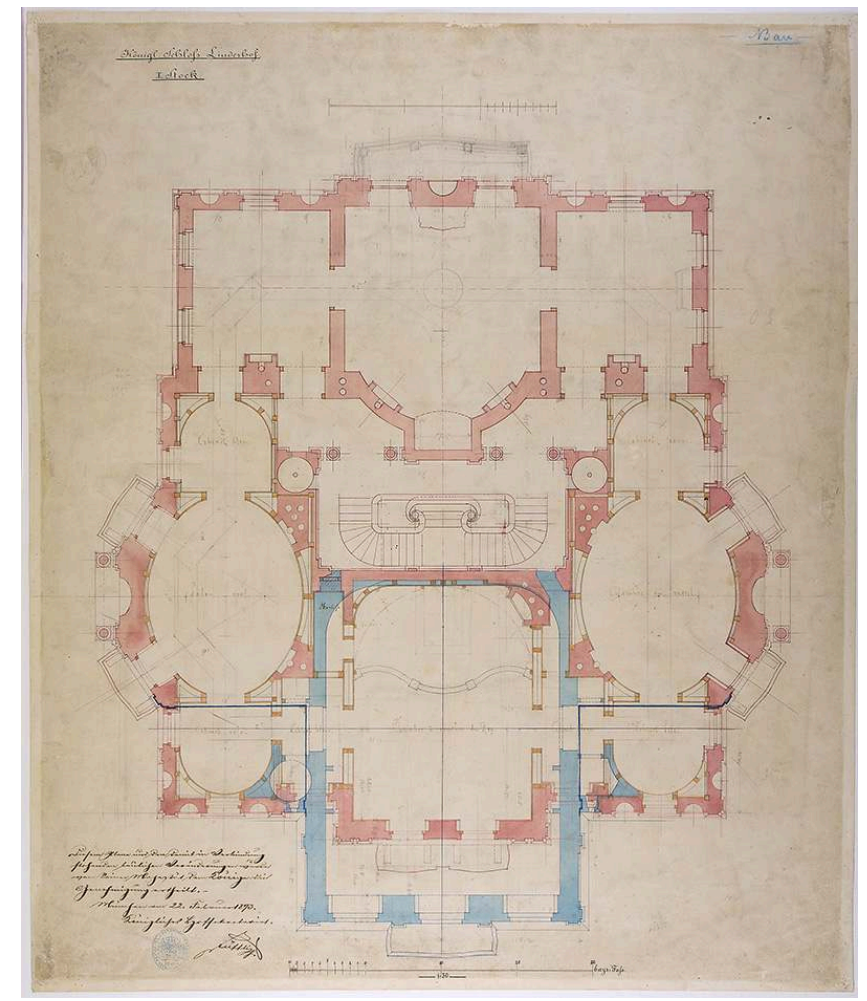

Plan du château de Linderhof, début 1873. Le plan est orienté au sud. La partie nord (inférieure) montre l'état de la construction, celle au sud l'achèvement prévu à la place de la petite maison du roi (approuvé le 22 février 1873). De plus, l'agrandissement de la chambre à coucher ordonné en 1884 est enregistré. BSV. L.II.-Mus. 1958.

(C) Bayerische Schlösserverwaltung. www.schloesser.bayern.de.

En 1873, on commença aussi à prévoir concrètement le remplacement de l'ancienne «petite maison du roi » par une nouvelle aile sud, qui devait également constituer la façade du petit château. Dès 1872, une version, qui ne fut pas réalisée, prévoyait de créer comme aile sud une « chambre de parade » entre un « salon de l'œil-de-bœuf » et une "salle des gardes $»^{2}$ - la référence à Versailles était donc aussi une des sources 
d'inspiration de Linderhof. Comme par la suite à Herrenchiemsee, la grande « chambre de parade" n'était sans doute pas conçue pour être utilisée comme chambre mais plutôt comme monument à Louis XIV, alors que Louis II voulait dormir dans la chambre à coucher rococo, plus petite ${ }^{3}$. À la place de ce projet, toutefois, à Linderhof on construisit comme pièce centrale du côté sud, en pendant de la chambre à coucher du nord, la salle des miroirs, sur le modèle du cabinet aux Miroirs des reichen Zimmer («chambres riches ») du palais de la Résidence à Munich ${ }^{4}$.

5 Après l'achèvement de la construction de l'aile sud, en 1877, le château de Linderhof resta inchangé et les ressources se concentrèrent sur le grand édifice de l'île du Chiemsee, dont la construction débuta en 1878. Les premiers projets pour le lit de la chambre de parade dédiée à Louis XIV furent prêts en 1876, les travaux s'achevèrent en 1883. Cette année-là furent réalisés les premiers plans pour le lit du petit appartement "Louis XV », achevé en 1886. En 1884, le roi ordonna d'agrandir et de réaménager complètement la chambre à coucher de Linderhof. Il ne vit pas l'achèvement de ce projet. Après sa mort, survenue le 13 août 1886, les châteaux de Louis II furent ouverts aux visiteurs et la chambre à coucher de Linderhof montrée dans son état inachevé ${ }^{5}$. Les pièces de mobilier réalisées et une partie des vieux meubles furent récupérées. Le reste de l'ancien mobilier, fut cédée en 1887 par le secrétariat de la Cour au Musée national bavarois. En 1968, il fut confié à titre de prêt permanent à l'Administration des châteaux de Bavière (die Bayerische Schlösserverwaltung), qui l'expose au musée Louis II du château de Herrenchiemsee.

\section{Les essais de reconstruction du lit de Louis XIV à Versailles par le roi Louis II}

6 Louis II avait commencé à s'intéresser aux chambres à coucher royales de Versailles avant de projeter ses constructions dans une solitude champêtre. Pour le nouvel aménagement de son appartement de la Résidence de Munich, en 1867, Louis exigea « ... le style fastueux, majestueux, comme celui qui dominait à la Cour de Louis XIV, et qui doit être le seul autorisé dans la réalisation de mes appartements... ${ }^{6}$. L'ébauche de la chambre à coucher reprend différents aspects de la chambre d'apparat de Versailles telle qu'elle se présentait depuis son aménagement de 1837, sous Louis-Philippe : une balustrade, un lit à baldaquin de forme analogue, placé dans l'axe, des tableaux de part et d'autre du lit, un buste du Roi-Soleil ${ }^{7}$. Ces éléments étaient connus par des documents iconographiques que Louis II faisait constamment rechercher par ses agents. Non seulement possédait-il la monographie de Jean Vatout (1792-1848) sur le palais de Versailles, premier volume de la série des "Souvenirs historiques des résidences royales de France $»^{8}$, de 1837 , et l'ouvrage de planches in-folio Souvenir d'une Promenade à Versailles, de 1838, mais aussi une photographie de la chambre d'apparat, qui montrait une disposition tout à fait similaire ${ }^{9}$. Ces vues semblent avoir servi de base aux artistes employés par Louis, car parmi les dessins et plans de ses projets de construction conservés par le Wittelsbacher Ausgleichsfonds (Fonds de compensation des Wittelsbach, Munich) et la Bayerische Schlösserverwaltung (l'Administration des châteaux de Bavière), on trouve une aquarelle qui copie la photographie et deux représentations individuelles $\mathrm{du}$ lit et de son environnement immédiat, qui reproduisent exactement sa structure, mais «corrigent " la composition ainsi que l'iconographie du décor textile (fig. 2). 
Figure 2

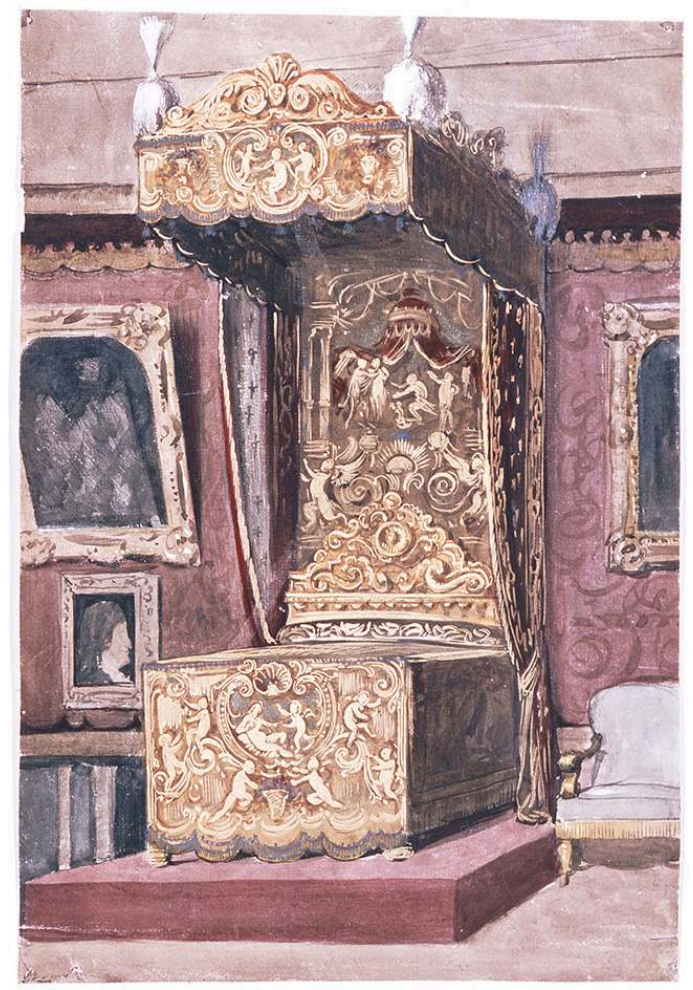

Premier essai de reconstitution du lit de Louis XIV de Simon Delobel, d'après l'état présenté en 1837 dans la chambre d'apparat de Versailles. On ignore quel artiste au service de Louis II exécuta ce dessin entre 1867 et 1869. Gouache avec rehauts d'or, 36 × 24,5 cm, WAF Inv.-Nr. B VIII 47.

(c) Wittelsbacher Ausgleichsfonds München.

7 Louis II voulait manifestement reconstituer le lit que Louis XIV avait utilisé de 1682 à 1701 dans le salon de Mercure et dont la description contemporaine, par de Soucy, avait été publiée par Vatout ${ }^{10}$. Il était considéré comme un sommet de l'art textile. Le tapissier de la Cour, Simon Delobel, passe pour y avoir travaillé douze ans. Louis II dut être très impressionné par la description puisqu'il la fit traduire en allemand ${ }^{11}$. On ne sait si Louis II s'était rendu compte que le lit installé dans la chambre d'apparat en 1837 et qui se trouve actuellement dans le salon de Mercure, contrairement à ce que Vatout affirmait, n'avait rien à voir avec le lit de Louis XIV, ou si, dans sa recherche des formes idéales, il avait accepté qu'il diffère de ce qui était considéré comme le lit d'origine ${ }^{12}$. Tous les états du projet de lit pour la chambre de parade du projet Versailles reprennent l'iconographie du lit exécuté par Delobel. Ils montrent de nombreux petits Amours et des représentations de Vénus et comme image principale, sur le dossier du lit, les nymphes coupant les ailes de l'Amour, sous un dais, dans un magnifique cadre architectural.

La conception des lits pour Linderhof et pour la chambre de parade de «Tmeicos Ettal» fut concomitante à ses débuts et les deux semblent avoir la même origine. Le premier essai déjà mentionné, qui conservait la forme du lit de Versailles de 1837, et n'en « reconstruisait» que le décor, fut vraisemblablement suivi, en 1869, d'un premier projet de lit que l'on peut clairement attribuer à Tmeicos Ettal et qui fait partie d'une série de feuilles très ornées, qui rendent exactement la description de Soucy: un premier projet de lit à quatre montants, suivi de deux dessins de lit à la duchesse ${ }^{13}$. Le 
lit a ici des formes droites, seul la forme du ciel est animée par un dôme et des tentures à festons trilobes et des galons. Les montants, dont le langage formel rappelle le style Louis XVI, devaient être richement tournés, sculptés et dorés, mais l'ornement le plus précieux était les tissus, qui devaient être stylistiquement plus proches du baroque tardif: les projets pour le dossier du lit, la courtepointe avec empiècements de soie, tentures et "bonnes grâces" ont un fond d'or et des broderies en relief d'or et d'argent, quelques-unes de couleur, très richement exécutées. Les rideaux ont des broderies assorties sur un fond rouge ${ }^{14}$.

\section{D'une forme rectangulaire à une forme sinueuse}

Un dessin de 1872 pour Tmeicos Ettal montre une chambre à coucher agrandie, bien différente de celle de Versailles. Le lit et le ciel aussi ont été remaniés; les formes rectangulaires sont remplacées par plusieurs courbes, les contours supérieurs et inférieurs des deux parties sont très sinueux et les montants antérieurs du lit, en forme de S, sont ornés de cariatides ${ }^{15}$.

Figure 3

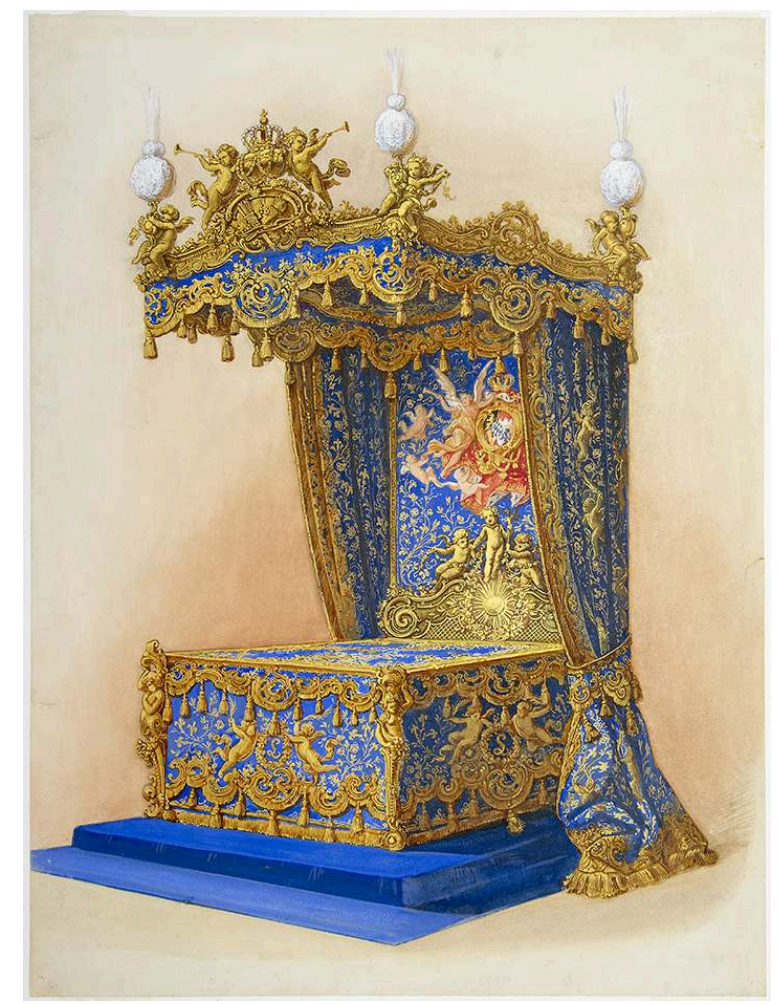

Premier projet pour le lit de la première chambre à coucher de Linderhof, Franz von Seitz, 1871. Gouache et rehauts d'or, 57 × $43 \mathrm{~cm}$. BSV, L.II.-Mus. 2522a.

(C) Bayerische Schlösserverwaltung. www.schloesser.bayern.de.

10 À Linderhof, on observe la même évolution. Le premier projet qui subsiste pour le lit est documenté par une feuille richement exécutée à la gouache avec des rehauts d'or (fig. 3), associée à un paiement de 88 florins à Franz von Seitz ${ }^{16}$, en 1871, pour le

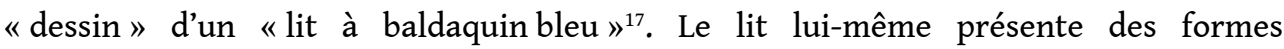
rectilignes, mais les angles antérieurs du soubassement, comme pour le lit de Tmeicos 
Ettal de 1872, sont formés par des termes, légèrement incurvés en forme de $\mathrm{S}$ : à gauche une figure masculine, à droite une figure féminine tenant une corne d'abondance. Les tentures de la courtepointe, montées sur un cadre ${ }^{18}$, sont étroitement liées à l'angle ainsi constitué. Le dais a un dessin galbé et ses contours supérieurs et inférieurs sont très sinueux. La bordure supérieure est sculptée, les tissus prévus ont des broderies très en relief, d'or exclusivement, sur fond bleu. Un seul motif a été repris directement des esquisses sur le thème de l'amour de Tmeicos Ettal: les armes de Cupidon, dans le cartouche central du ciel (carquois, arc et torche), qui figuraient, selon la description, en deux endroits du lit de Louis $\mathrm{XIV}^{19}$. Au-dessus, deux putti soufflant dans une trompette présentent sur un coussin la couronne royale de Bavière avec les colliers d'ordre. Sur chaque face du tour de lit, deux putti tiennent l'initiale du roi, Louis XIV ou Louis XV, dans une couronne de fleurs; d'autres amours brodés, voletant entre des rinceaux, peuplent les côtés extérieurs des rideaux. L'intérieur des courtines ne présente que des tiges de lys ondulantes ${ }^{20}$. Le dossier du lit a pour motif principal les armes de Bavière, sur un écu rouge, portées par deux Génies soufflant dans une trompe. L'élément sculpté qui couronne la tête de lit peut être décrit comme un fronton incurvé, au centre duquel rayonne un soleil - motif du lit installé en 1837 à Versailles, non décrit par Soucy et pourtant repris par Louis II sur le lit de la Résidence et les quatre lits d'Herrenchiemsee et Linderhof. À Linderhof, deux putti avec des guirlandes de fleurs sont assis sur le bord supérieur, encadrant un autre putto, debout, qui tient un lys.

11 Le principe général de la composition et du décor du lit est une file de volutes qui souligne le bord inférieur et supérieur des tentures de la courtepointe sur les faces latérales et antérieure (au pied du lit) ainsi que les bordures supérieure et inférieure du dais. Le bord supérieur du dais est sculpté et chacun des trois côtés a en son centre une partie relevée en forme d'arc et les trois bandes correspondantes forment des lambrequins. Les volutes pendantes, avec des acanthes et des ornements rocaille, devaient être exécutées en broderie en relief, chacune pourvue dans sa partie inférieure d'une épaisse frange dorée et garnie de nombreux glands dorés. 


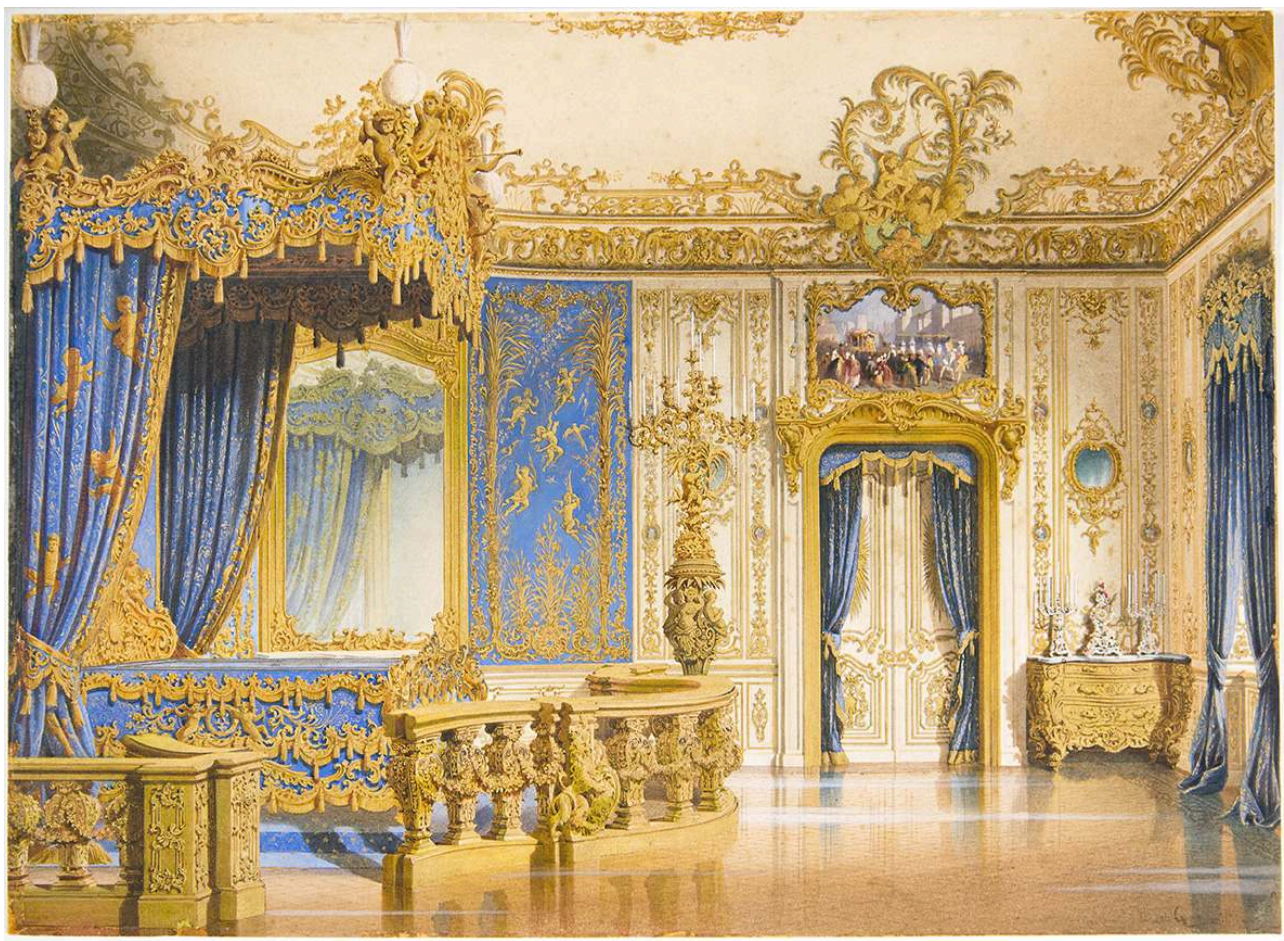

Vue de la première chambre à coucher du château de Linderhof, signée et datée, Angelo Quaglio, 1871. Aquarelle et rehauts d'or, 39 × 53,5 cm. BSV, L.II.-Mus. 265.

(c) Bayerische Schlösserverwaltung. www.schloesser.bayern.de.

12 En 1871, le scénographe Angelo II Quaglio (1829-1890) fit un dessin de la pièce (daté et signé), qui montre le lit dans une forme légèrement différente (fig. 4) ${ }^{21}$. De cet état, nous avons également une ébauche du lit seul, liée à un paiement de 90 florins, en 1871, pour le «dessin d'un bois de lit avec ciel de lit $»^{22}$ attribué depuis longtemps à Adolf Seder (1842-1881) ${ }^{23}$ mais resté inédit (fig. 5). Ce n'est que maintenant que l'on a prêté attention à l'inscription contemporaine, évidente : « Nouveau dessin de lit par Seder. Baldaquin et bois de lit (devant) incurvés. Il y aura des étoiles brodées à la marche du lit ». La principale différence avec le projet précédent est que l'extrémité du lit dépasse à présent de la courtepointe, tendue sur un cadre et fixée entre les montants du lit, audessus du matelas. Celle-là n'a d'ailleurs plus que des pentes sur les côtés, et non plus au pied du lit. Le pied du lit se compose maintenant d'un cadre à peu près ovale et d'une garniture en tissu et interrompt la structure générale horizontale apportée, dans le projet précédent, par la frise de volutes qui l'entourait. Le pied du lit domine ici l'ensemble du lit, dont l'aspect de meuble textile laisse place à celui de meuble sculptural. Les formes du ciel de lit sont aussi plus sinueuses, tant dans le plan que dans la ligne de contour supérieure, qui ne se compose plus que d'arcs, alors que dans le projet précédent, elle comportait à la fois des sections droites et des sections légèrement courbes. L'agencement en frises horizontales est toujours là, mais interrompu sur le devant, il cesse de dominer. 
Figure 5

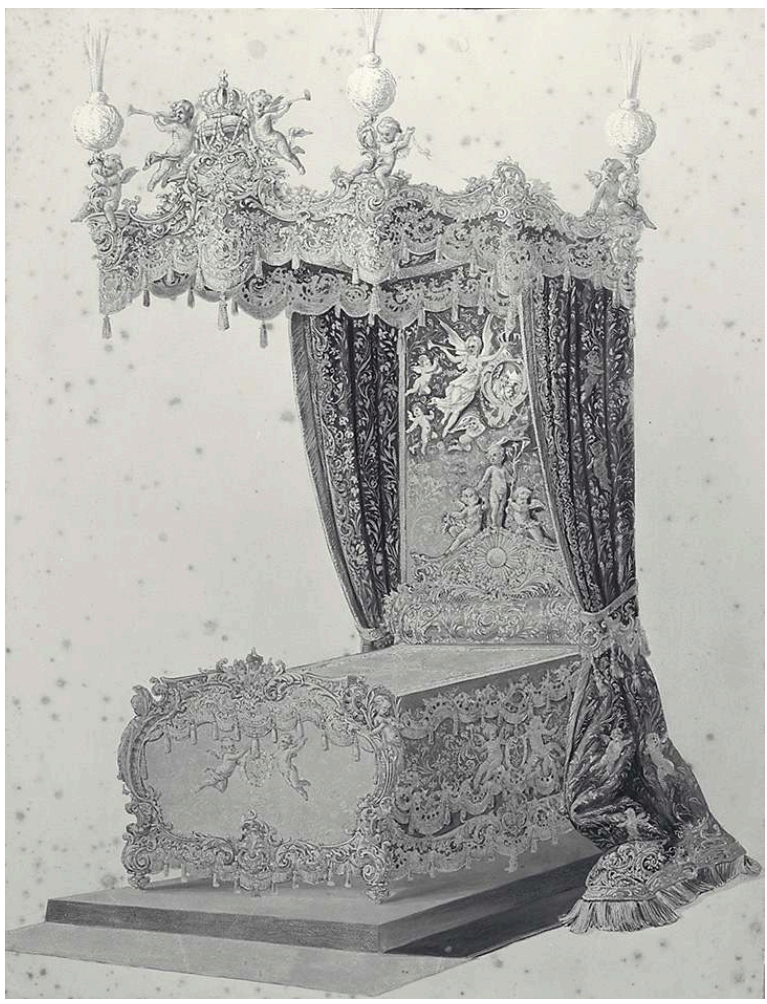

Deuxième projet pour le lit de la première chambre à coucher de Linderhof, Adolf Seder, 1871. Gouache avec rehauts d'or, 59 × 45,5 cm. WAF Inv.-Nr. B VIII 39.

(C) Wittelsbacher Ausgleichsfonds München.

Les variantes entre le dessin de Seder et l'exécution, «travaux de menuiserie et de sculpture d'ornements " par la maison Anton Pössenbacher, sculpture de personnages de Johann Hirt, dorure par la maison Josef Radspieler, broderies de l'atelier Mathilde Jörres et des maisons Alckens et Bornhauser, paiements en 1872, reliquats en $1873^{24}$, sont si minimes qu'on ne peut considérer qu'il y eut d'autres projets (fig. 6). On ne remarque que le remplacement des rocailles, qui forment le contour des lambrequins, par des feuilles en forme d'acanthes; dans la réalisation, les rocailles sont placés, isolément, à l'extrémité du lit et sur les bordures antérieures et inférieures des grandes tentures ainsi que dans les bordures du panneau mural. 


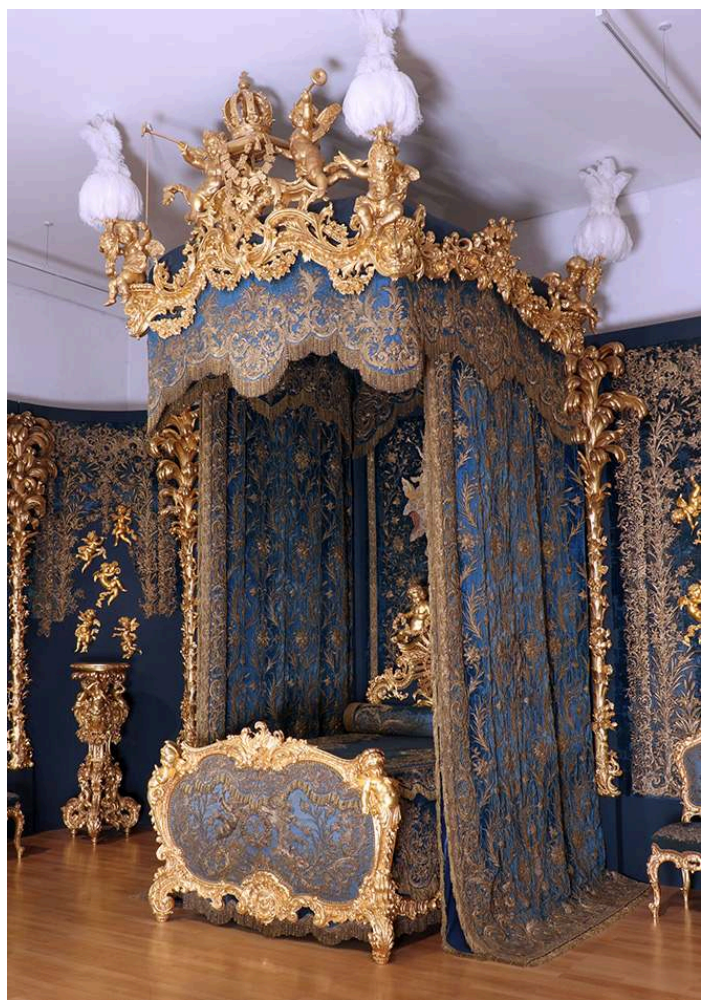

Vue d'ensemble du lit, état en 2018.

Phot. Sybe Wartena. (c) Bayerisches Nationalmuseum.

14 Le changement de ton, qui intervint en 1871 à Linderhof, peut être comparé avec le remplacement qui eut lieu au plus tard en 1872 du lit à montants droits par un lit sculptural pour Tmeicos Ettal. Mais la question de la succession chronologique ne peut toutefois pas être élucidée, faute de documents datés ${ }^{25}$. Ceci s'applique également à la deuxième modification de plan, qui fut réalisée à peu près à la même période dans les deux projets : l'ajout de palmiers, de la hauteur de la pièce, sculptés et dorés, comme séparation entre les panneaux du mur de l'alcôve. Une esquisse de panneau pour Linderhof ${ }^{26}$ porte la correction suivante, du 12 août 1872, de Lorenz von Düfflipp (1821-1886), secrétaire de la Cour, qui servait d'habitude d'intermédiaire entre Louis et ses artistes : "le mur de fond de la chambre à coucher doit être entièrement tendu de velours bleu, y compris les bandes qui séparent les différents champs »-y figure le nouvel arrangement de palmiers sur un revêtement mural continu (fig. 7). 


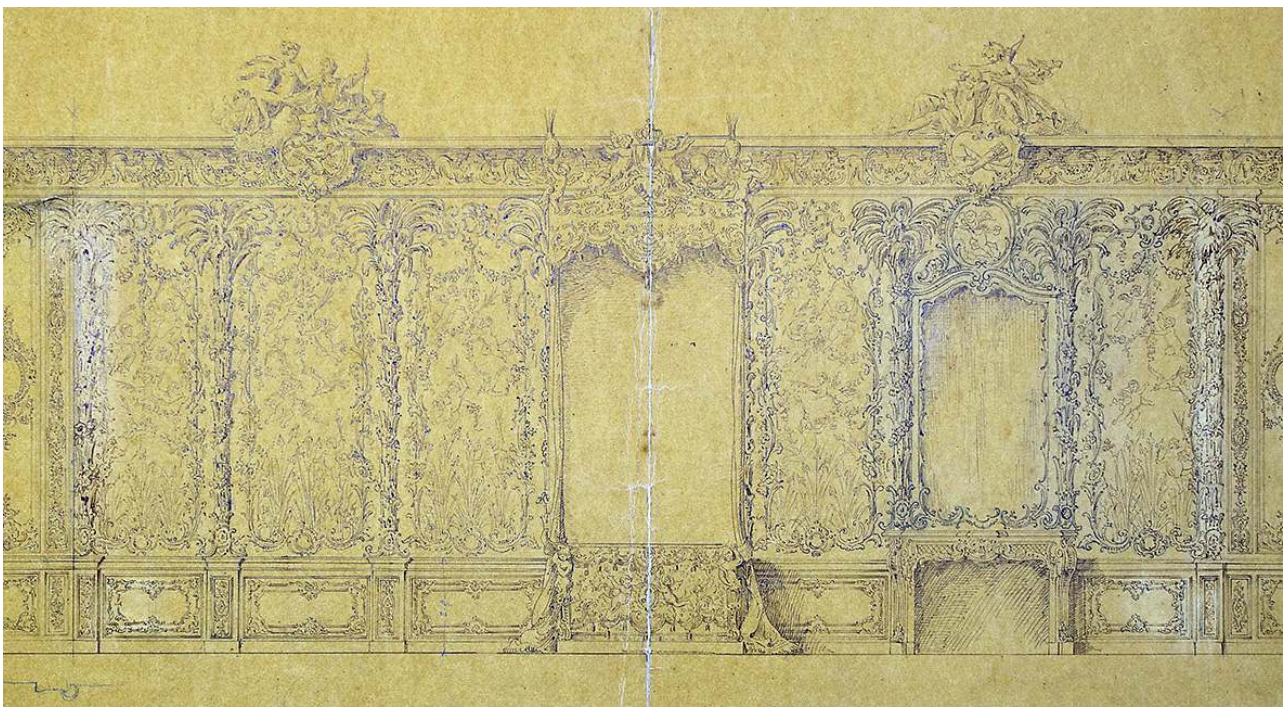

Projet de la première chambre à coucher de Linderhof, élévation, Georg Dollmann, 1872. Calque, découpe. BSV, L.II.-Mus. 2000/2.

(c) Bayerische Schlösserverwaltung. www.schloesser.bayern.de.

15 Jusque-là, les projets prévoyaient de séparer les "panneaux » textiles au moyen de lésènes blanches avec une ornementation dorée (fig. 8). Les projets suivants prévoient, à côté des palmiers, de modifier sensiblement les « panneaux » de broderies : les touffes de lys, sur les côtés, qui à leur tour rappellent les palmiers, et les bordures, qui étaient disposées dans le dessin comme une demi-feuille, devaient être remplacées par un fin bâton ornementé d'un rinceau de fleurs. Les tiges de lys et la petite bordure, cette dernière sous forme de rangée de feuilles, furent tout de même exécutées. En 1872, Adolf Seder fut payé pour le dessin d'un palmier et des modifications aux broderies des panneau $x^{27}$, et leur exécution fut acquittée en 1873. Ces modifications - ajouts à une ornementation moins dense - sont clairement perceptibles (fig. 9). 
Figure 8

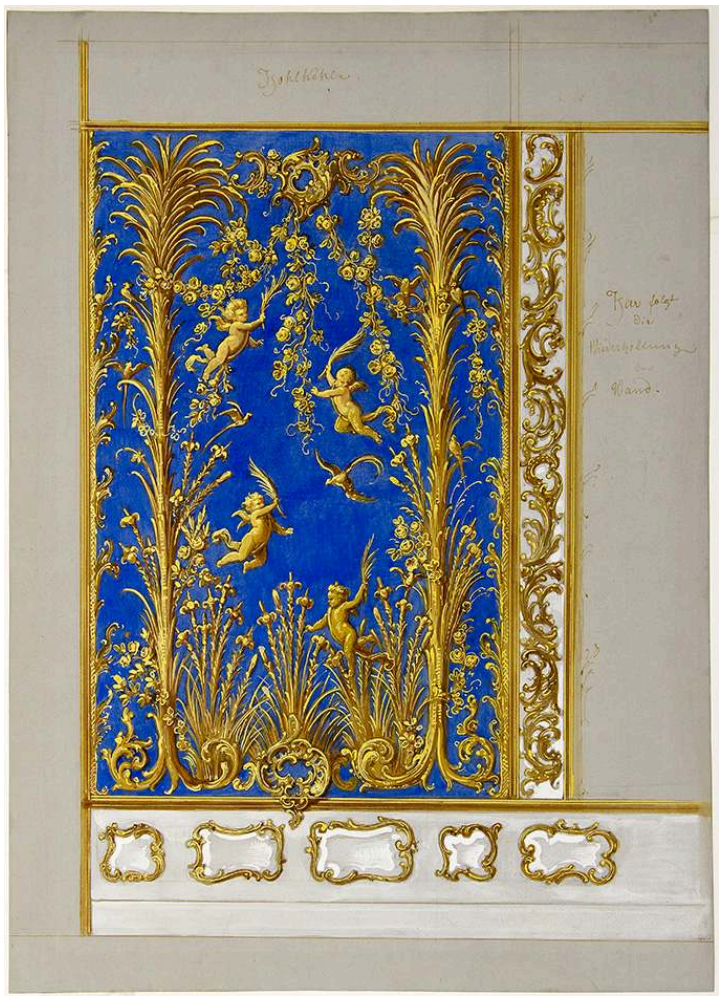

Projet d'un panneau mural de l'alcôve de la première chambre à coucher du château de Linderhof Franz von Seitz, 1871. Gouache avec rehauts d'or, 55,3 x 39,5 cm. BSV, L.II.-Mus. 2029.

(c) Bayerische Schlösserverwaltung. www.schloesser.bayern.de. 


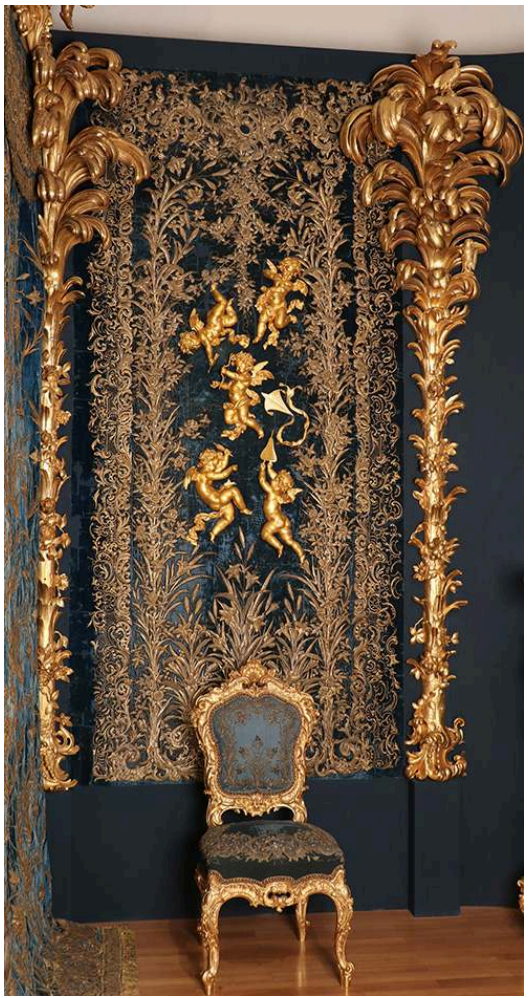

Panneau mural, à droite du lit, état en 2018.

Phot. Sybe Wartena. (c) Bayerisches Nationalmuseum.

Les palmiers ont été rapprochés de façon convaincante par Beate Goertz des palmiers des encadrements de loges au théâtre de la Résidence, à Munich, dus à l'architecte François de Cuvilliés (1695-1768), vers 1751-1755 ${ }^{28}$. On pourrait donc y voir un indice de l'invention du motif pour Linderhof, puisque si l'on doit rechercher les modèles pour le projet Versailles dans l'art de la construction et de l'aménagement de la Cour de France et non chez les Wittelsbach de Bavière, pour Linderhof, la référence est bien Munich. D'autre part, on se donna encore plus de mal dans les études pour Tmeicos Ettal que pour Linderhof, comme le montre par exemple le fait que le dernier dessin fut même peint à l'huile ${ }^{29}$. Mais on ne peut pas en conclure absolument que Linderhof ait servi à l'occasion de terrain d'expérimentation, où l'on testait la faisabilité des idées du grand projet de Tmeicos Ettal.

\section{Origines de l'ébauche du lit rococo}

17 Grâce à une note dans les livres de caisse du cabinet, on peut retracer, au moins sommairement, la nouvelle tendance du lit. Des récépissés de paiement au tapissier Max Steinmetz indiquent : "Pour la confection d'un lit de prestige de style Louis XV, d'après un croquis du directeur Franz [von] Seitz, ce dernier revu par le soussigné "Steinmetz", sur commande du secrétariat royal de la Cour, d'après une œuvre de Meissonier (sic), et exécuté d'après ces dessins approuvés par Sa Majesté ${ }^{30}$.» Le dessin de Seitz ne correspond donc pas aux conceptions que se faisait le roi du rococo Louis XV. Il semble davantage avoir eu à l'esprit un style comme celui qu'incarnait Juste-Aurèle Meissonnier. Louis II avait en sa possession l'œuvre de cet artiste, publié à 
Paris vers 1750, avec un grand nombre de ses dessins de pièces d'orfèvrerie, de mobilier, de décors intérieurs et d'architecture. Dans l'exemplaire conservé aujourd'hui à la Bibliothèque nationale de Bavière ${ }^{31}, 17$ planches portent des annotations se rapportant à des projets de construction et d'aménagement de Louis II. Ainsi, le projet d'ensemble pour le cabinet de travail, achevé en 1872, peut être rapproché, sur la base de l'annotation "Cabinet de travail, vert », de la planche 85, "Projet du sallon de la princesse Satorinski [Czartoryski] en Pologne», et un "miroir provisoire pour la chambre à coucher ", de la planche 87 , «Cabinet pour le comte Bielinski ». Aucune de ces annotations ne se rapporte au lit, mais le langage formel de ses sculptures semble être inspiré par le style de Meissonnier. C'est ce que l'on voit dans des motifs comme les rocailles rebondies, des volutes marquées, compactes, ou des sections à peine galbées, ornées d'une fine bordure rocaille, alors que la partie accolée est à godrons. La combinaison de rocailles et de feuilles d'acanthe ou la tresse de sépales sur le fût des figures d'angelots engainées, aux angles, se retrouve aussi chez Meissonnier ${ }^{32}$. Les pieds remarquablement massifs, qui semblent organiquement appartenir au châssis inférieur en lieu des poteaux d'angle (fig. 10) rappellent fortement la partie supérieure de son dessin de console de la planche 48 , «Table d'appartement ${ }^{33}$.

Figure 10

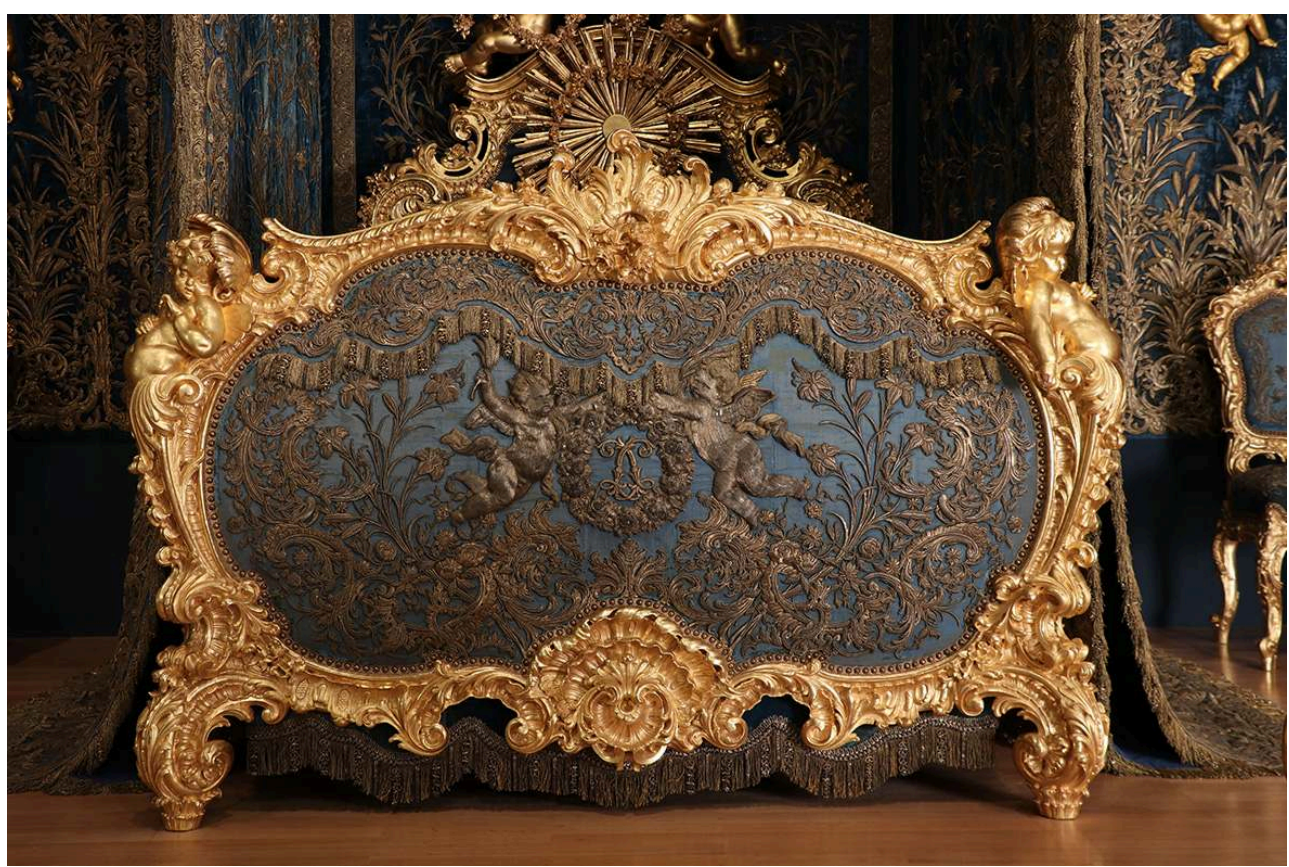

Extrémité du lit, état en 2018

Phot. Sybe Wartena. (c) Bayerisches Nationalmuseum.

Les motifs ornementaux pourraient aussi avoir d'autres sources, Meissonnier n'étant sûrement qu'une possibilité. Il est toutefois important de constater que le lit, dans son ensemble, ne peut se fonder sur aucun modèle du xvIII siècle, qu'il s'agisse d'un meuble existant ou d'un dessin. On ne connaît aucun ciel de lit ou lit du $\mathrm{xVIII}^{\mathrm{e}}$ siècle qui présente une sculpture aussi somptueuse, et en tout cas, associée à des tissus

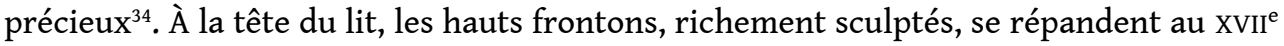
siècle, mais il n'y a pas de pied de lit élevé. Les pieds « renversés » ou les termes qui somment l'angle des montants galbés rappellent certaines commodes ou consoles 
rococo, mais il est exclu qu'elles aient inspiré les montants dans leur ensemble. On y voit davantage le souvenir de cartouches, dont bon nombre, très proches, sont dus à des dessinateurs du XVIII ${ }^{\mathrm{e}}$ siècle, et en particulier à Meissonnier ${ }^{35}$. Il faut souligner ici que le lit de la chambre de parade, à Herrenchiemsee, malgré une disposition très similaire, reprend dans le langage des formes des éléments de style des décennies de la fin du XVII et du début du XVIII ${ }^{e}$ siècle. Le lit du petit appartement, par contre, est étroitement apparenté à celui de Linderhof, y compris dans le détail des ornements.

\section{Fonction du lit et iconographie}

19 La hauteur du pied de lit attire l'attention sur une caractéristique qui ne serait pas concevable au XVIII ${ }^{\mathrm{e}}$ siècle. Les côtés du lit sont remarquablement bas (fig. 11) ${ }^{36}$. Le bord supérieur du matelas ne peut pas avoir été beaucoup plus haut que les parties supérieures du côté galbé et chantourné. Entre le matelas et la courtepointe montée sur un sommier à lattes et suspendue entre la tête et le pied du lit, il restait un espace considérable. Ceci se voit pour des lits Louis XVI et Empire ${ }^{37}$, dont l'extrémité inférieure est à peu près aussi haut, que le chevet, mais pas pour des lits rococo, où de tels couvrelits sont posés sur le matelas. En ce point ce n'est pas clair comment l'extrémité inférieure devait être décoré en ces ébauches, qui prévoyaient cette partie basse : est-ce que le décor appartenait aux tentures de la courtepointe ou est ce qu'il devait faire partie du pied de lit? Le Lit exécuté pour Linderhof semble d'indiquer le dernier, car les lambrequins supérieurs des pentes latérales se prolongent dans le décor au châssis de l'extrémité inférieure.

Figure 11

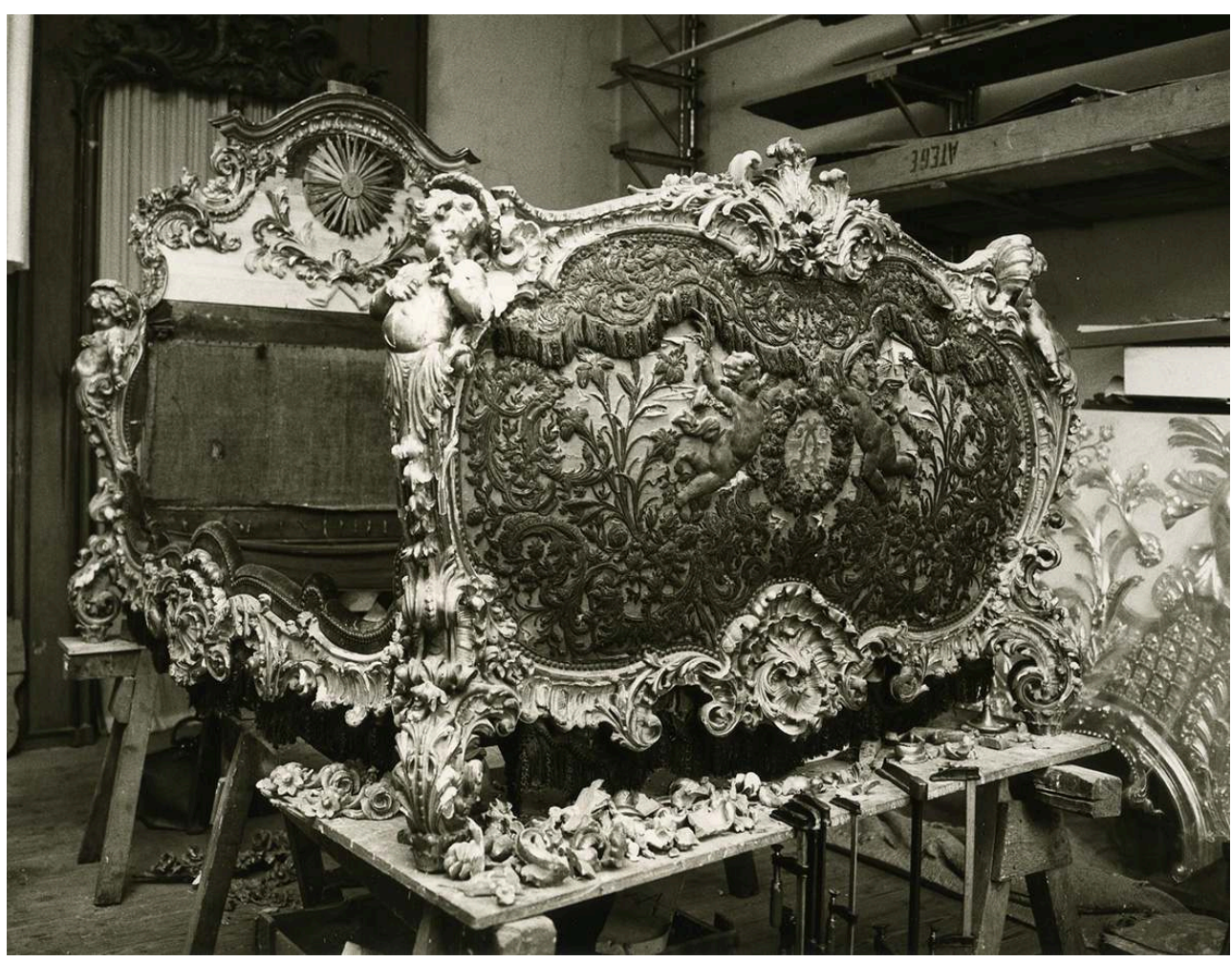

Bois de lit, état avant 1968.

Phot. Bayerisches Nationalmuseum. (c) Bayerisches Nationalmuseum. 
concept technique et fonctionnel du XIX $x^{e}$ siècle avec des formes structurelles et décoratives d'époques plus anciennes est typique de nombre de pièces réalisées pour Louis II par des artisans d'art, qu'il s'agisse de calèches et de traîneaux ou de systèmes de chauffage et d'installations téléphoniques dans ses châteaux. C'est ainsi que le lit, comme les deux autres à Herrenchiemsee, était équipé d'un matelas de 60 livres de crin de cheval, en trois parties, et d'un « matelas de Federn [ce mot vouloir dire aussi bien plumes que ressorts], rembourré de sangles, de Federn, de crin de cheval et de diverses toiles de lin... ${ }^{38}$, sans doute indiquant un sommier à ressorts.

21 Le programme iconographique du premier lit de Linderhof est bien moins vaste que celui de chacun des lits d'Herrenchiemsee, mais on peut établir une parenté. Sur le lit lui-même ne sont représentés que l'initiale de Louis, la couronne de Bavière avec les ordres de chevalerie, des putti voletant parmi les rinceaux sur les faces des tentures et, au centre du ciel de lit, les armes de Cupidon (fig. 12).

Figure 12

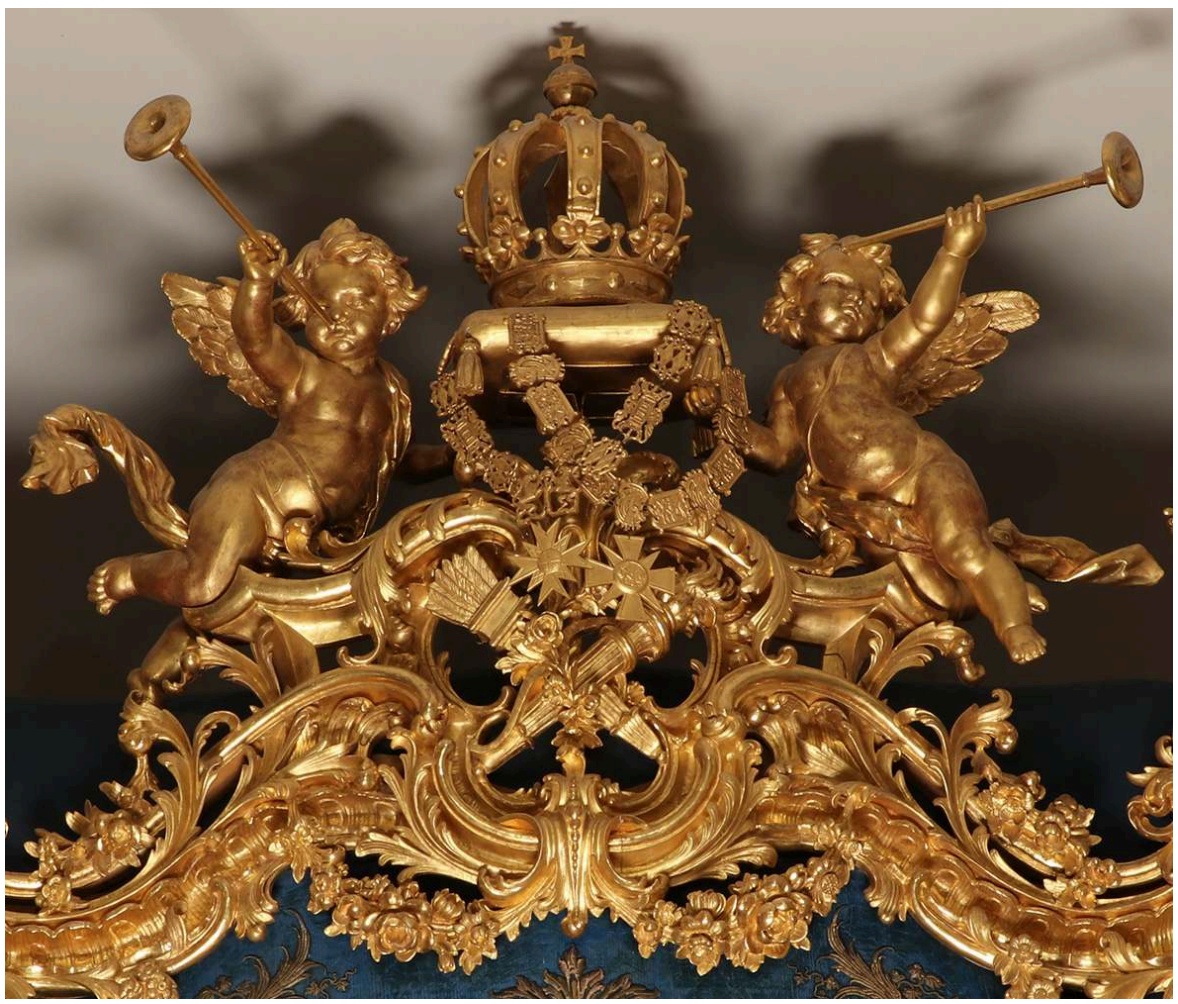

Ciel de lit, partie centrale de la face antérieure, état en 2018.

Phot. Sybe Wartena. (c) Bayerisches Nationalmuseum.

Les panneaux muraux attenants, de velours bleu, avec leurs bordures rocaille, leurs touffes de lys et guirlandes de roses brodées d'or, forment chacun un cadre précieux pour cinq putti de bronze doré voletant ${ }^{39}$. On considère de façon convaincante que ces putti ont pris pour source d'inspiration formelle les décors muraux de la salle de repos du pavillon d'Amalienburg, dans le parc du château de Nymphenburg ${ }^{40}$, mais à la différence de ceux-ci, ce ne sont pas les quatre éléments qui ont été choisis. Leur expression peut être décrite comme vive et espiègle. Certains font de la musique avec des cymbales, de minuscules violons et trompettes, d'autres font voler un cerf-volant, jouent avec un perroquet ou dansent. Ils s'assemblent parfaitement avec les groupes de 
personnages des moulures du plafond, cinq couples mythologiques exécutés en stuc doré par le sculpteur Franz Walker, en 1871: Amour et Psyché, Bacchus et Ariane, Vénus et Mars, "Diane regardant Endymion endormi " et "Vénus cajôle Adonis " ${ }^{41}$. L'ordre de Louis II du 12 août 1872 selon lequel « tout ce qui a un rapport avec l'amour (au sens mythologique) doit être dans la chambre à coucher $»^{42}$ semble en être une confirmation ultérieure. Le lit de la chambre de parade, à Herrenchiemsee, ne présentait pas moins de 23 scènes sur le thème de la puissance de Vénus, le lit de la chambre à coucher du petit appartement comporte à son extrémité, sur les poteaux, Vénus et Adonis, puis le repos de Vénus en relief, et sur le dossier du lit, le triomphe de Louis XIV sur le vice.

L'ornementation brodée des panneaux muraux, dont le motif principal est le lys, symbole de pureté, peut avoir été conçue pour faire contraste avec l'ambiance suggérée par les groupes d'amoureux, les putti ou les Cupidons. L'iconographie du ciel de lit va dans ce sens encore plus clairement: c'est la colombe du Saint-Esprit tenant une ampoule dans son bec qui est ici représentée (fig. 13).

Figure 13

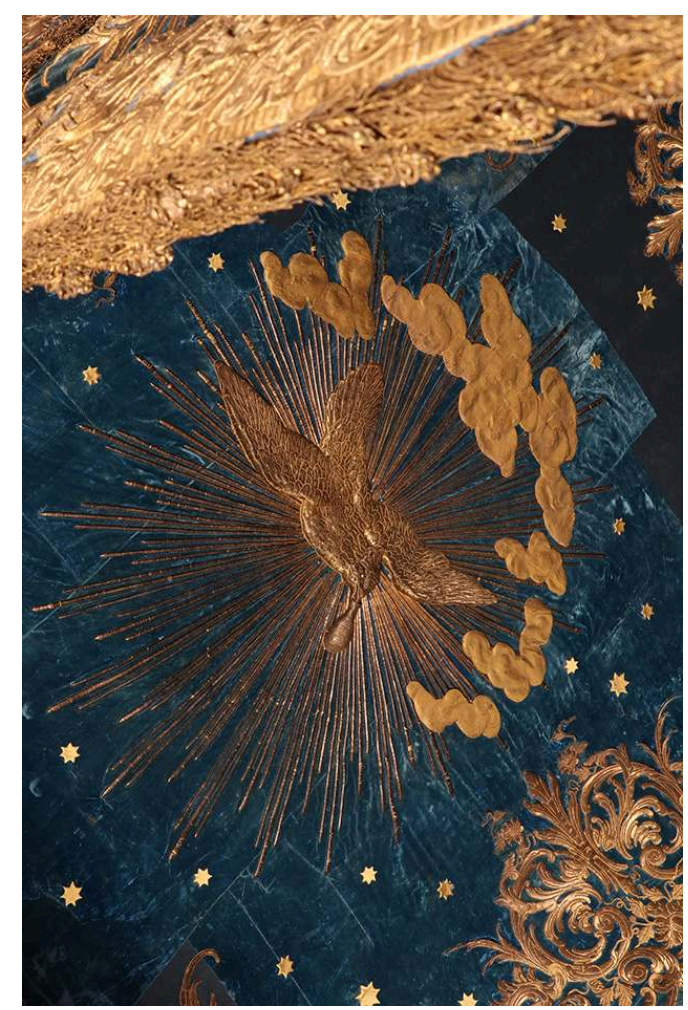

Ciel de lit, dessous : la colombe du Saint-Esprit avec l'ampoule d'huile sainte (en haut de l'image, courtines de droite), état en 2018

Phot. Sybe Wartena. (c) Bayerisches Nationalmuseum.

Ce motif se retrouve en 1884 à Herrenchiemsee sur le lit du petit appartement ${ }^{43}$. Entre 1875 et 1881, il apparaît de nouveau sur le dossier du lit de la grande chambre de parade d'Herrenchiemsee. Cette scène, Le Vou de Louis XIV, réalisée dans une technique appelée peinture à l'aiguille, a pu être installée pour masquer celle des Nymphes coupant les ailes de l'Amour ${ }^{44}$. Elle montre Louis XIV dédiant sa couronne à la Vierge, pendant que l'Enfant Jésus reçoit l'ampoule d'huile sainte que lui apporte en volant la 
colombe du Saint-Esprit ${ }^{45}$. Que l'espérance d'un appui divin dans son combat pour une royauté idéale ait été un thème important pour Louis II, les notes de son journal, entre autres, le montrent bien. Dans sa chambre à coucher de la Résidence de Munich, déjà, était accroché à côté de son lit un tableau du sacre et du couronnement de Louis XIV, sacre dans lequel Louis II voyait bien une sanctification d'une royauté voulue par Dieu. Le thème de l'onction est d'ailleurs une constante dans les chambres à coucher «françaises" du roi Louis, et la colombe avec l'huile sainte est pour la première fois apparue sur le lit de Linderhof.

\section{L'abandon de la première chambre à coucher de Linderhof et son histoire muséale}

En 1884 , le roi Louis II fit agrandir la chambre à coucher de Linderhof ${ }^{46}$. Dès le premier projet, il est clair que l'on cherchait à faire référence à la chambre à coucher des reichen Zimmer de Charles-Albert ${ }^{47}$ à la Résidence de Munich, parce que l'on y a pris les mesures du lit, et qu'elles ont été communiquées, les dimensions de la chambre à coucher des reichen Zimmer et celles de la première chambre à coucher de Linderhof pour les comparer ${ }^{48}$ ainsi que celles des deux lits et de leurs ciels de lit ${ }^{49}$. De plus, Louis II avait ordonné que la disposition des fenêtres et des portes soit calquée sur celle de la chambre à coucher des reichen Zimmer et «que les broderies des murs soient comme celles de la chambre à coucher des reichen Zimmer $\aleph^{50}$. Si les lambris sculptés et les stucs du plafond présentent une concordance plus étroite, les revêtements muraux adoptent les mêmes lignes directrices. En effet, les dimensions du deuxième lit, de 255 par $300 \mathrm{~cm}^{51}$, avoisinent celles du lit des reichen Zimmer, qui, selon les mesures de 1884, faisait environ 238 par $311 \mathrm{~cm}$, alors que la surface du premier lit ne fait que 180 par $243 \mathrm{~cm}$. Alors que les travaux avaient déjà bien commencé, arriva l'ordre pour «le plafond du ciel de lit, et aussi la colombe avec l'ampoule d'huile, les rayons, les étoiles et les nuages $\aleph^{52}$, qui soulignaient encore l'importance de ce programme iconographique.

Figure 14

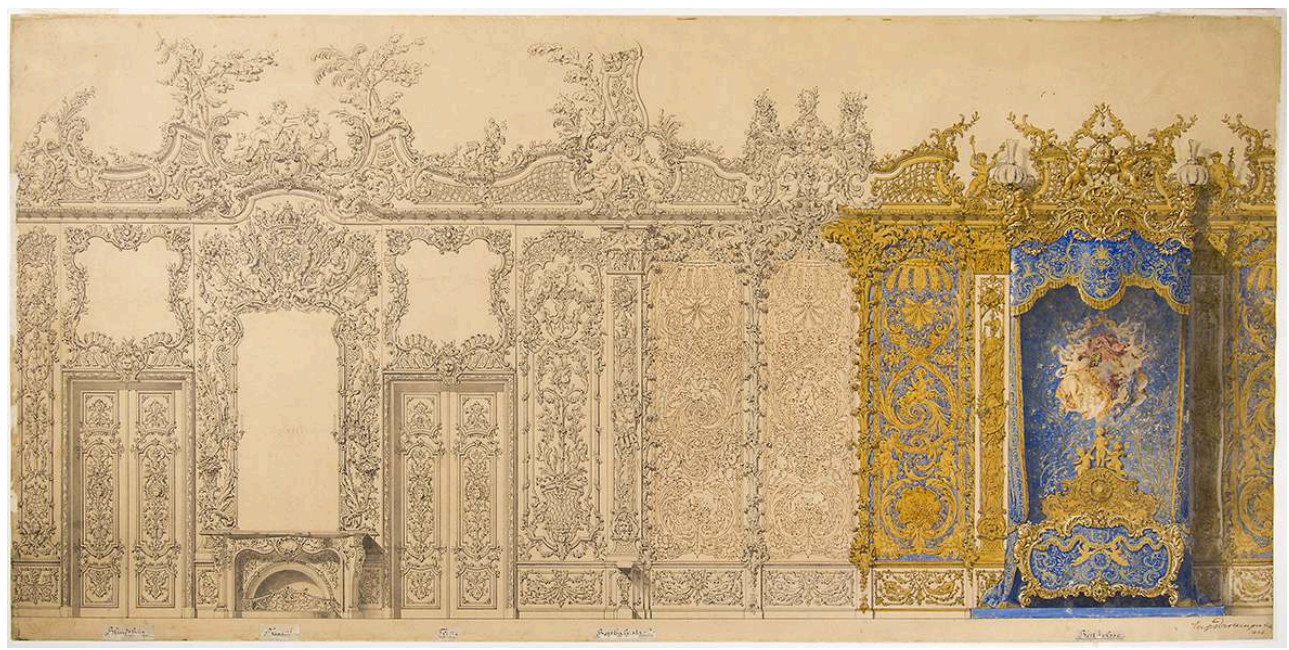

Projet pour la deuxième chambre à coucher de Linderhof, élévation. Eugen Drollinger, 1884. Plume, rehauts d'aquarelle, 45,3 × $92 \mathrm{~cm}$. BSV, L.II.-Mus. 268.

(C) Bayerische Schlösserverwaltung. www.schloesser.bayern.de. 
On n'a pas conservé de projet individuel pour le lit ${ }^{53}$, bien que l'on rapporte que des dessins aient été soumis au roi pour approbation ${ }^{54}$. La seule feuille connue qui y a trait, de l'architecte Eugen Drollinger (1858-1930), en $1884^{55}$ (fig. 14), correspond dans presque tous ses détails, proportions exceptées, au premier lit réalisé. La seule différence notable est la traverse supérieurepied, plus galbée, formée, des deux côtés du rinceau d'acanthe central, de deux courbes très marquées au lieu d'une seule, plus fine et étirée. Et c'est précisément à cet endroit, dans l'exécution du deuxième lit, que s'observe la plus grande différence avec le projet de Drollinger, instruit par « les ordres suprêmes, en ce qui concerne l'aménagement de la chambre à coucher de Linderhof ", transmis par l'architecte Julius Hofmann au valet de chambre Lorenz Mayr le 27 mars 1886, selon lesquels « la couverture du lit devait pendre par devant et sur les côtés » ${ }^{56}$. Pour obtenir ce résultat - aucune autre explication ne semble plausible - la bordure supérieure du pied a été radicalement modifiée : au lieu des courbes très marquées avec des feuilles d'acanthe fournies ou des rocailles, qui devaient largement dépasser les têtes des putti qui sont sur les côtés, le pied de lit a été coupé ras à la hauteur des têtes de putti et la frise remplie de petits éléments d'ornement, ce qui, vers le bas, donne une ligne mouvementée, fragmentée (fig. 15).

\section{Figure 15}

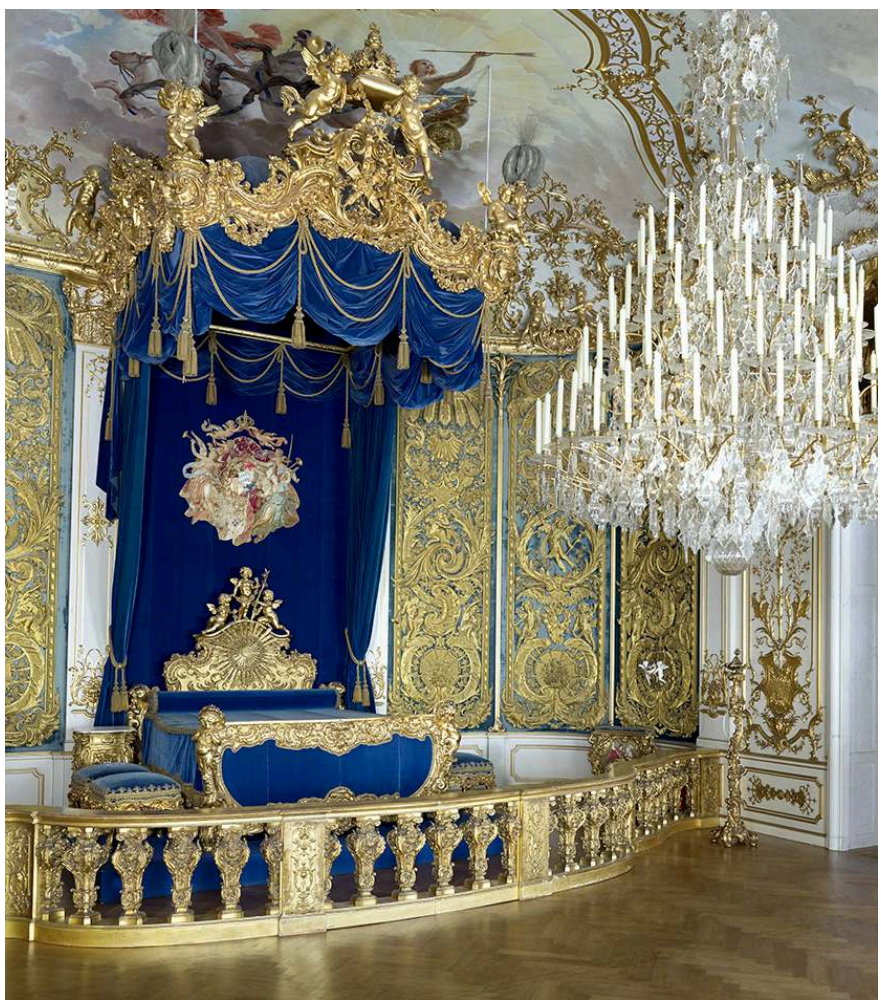

Deuxième chambre à coucher de Linderhof, état en 2018.

(c) Bayerische Schlösserverwaltung. www.schloesser.bayern.de.

Cette solution peu heureuse, qui, outre la terminaison supérieure, affecte les proportions du pied de lit, si important, aurait certes été masquée si la courtepointe avait été réalisé avec des tentures non seulement sur les côtés (ce qui fut exécuté) mais aussi, conformément aux ordres, à l'extrémité du lit ; mais les sculptures se dressent en avant, loin des traverses des extrémités, de sorte qu'il aurait fallu recourir à au moins 
une autre construction pour que les tentures pendent librement. Cet effet peu satisfaisant est encore accentué par le fait que, pour des raisons d'économie, la garniture est de simple velours bleu et que l'on a renoncé aux broderies d'or. Avec des incertitudes, selon les états du projet de 1871-1872, sur la hauteur du pied de lit, et sur la question de savoir si la courtepointe devait ou non comporter aussi une tenture à l'extrémité du lit, on a l'impression que l'on ne mesura pas, dans les échanges entre le roi et les artistes et artisans chargés de l'exécution, les conséquences de ce point ${ }^{57}$.

Le 24 octobre 1887, soit 16 mois après la mort du roi Louis II, le lit, tous les tissus, le ciel démonté en 18 morceaux, les tentures murales, les quatre chaises et deux tabourets qui allaient dans la chambre à coucher, ainsi que les rideaux et les portières, furent remis par le Secrétariat de la Cour au Musée national bavarois, qui exposa aussitôt cet ensemble dans la salle 23, au premier étage de l'ancien bâtiment du musée, dans la Maximilianstrasse ${ }^{58}$. Une partie du cadre du ciel de lit, manquant, fut retrouvé sur une indication du conseiller royal Ludwig Peter von Klug, de l'administration des biens royaux, au musée des Arts décoratifs de Strasbourg, qui avait acquis quelques objets provenant de la succession de Louis II, et fut récupéré fin 1887. Dès 1888, le lit fut présenté à l'Exposition nationale allemande des arts décoratifs, à Munich, dans un pavillon consacré à Louis II ${ }^{59}$ et en 1893 , montré à l'Exposition universelle de Chicago, dans la salle de l'Association bavaroise des arts décoratifs ${ }^{60}$. Suivit une nouvelle acquisition, le 29 juillet 1894 : «six palmiers sculptés et dorés provenant de l'ancienne chambre à coucher du château de Linderhof, de l'administration des biens du roi Otto » ${ }^{61}$.

Dans le nouveau bâtiment du Musée, dans la Prinzregentenstrasse, inauguré en 1900, la salle 48 était consacrée au roi Louis II. À côté des éléments de la chambre à coucher figuraient le célèbre portrait officiel de Louis II à vingt ans par Ferdinand von Piloty (1828-1895), un buste de Richard Wagner et quelques autres objets. Il faut noter l'intégration architecturale, qui citait le salon de l'œil-de-bœuf, et, de chaque côté du lit, introduisait des axes de miroirs mais renonçait à des caractéristiques comme l'alcôve arrondie ou la balustrade incurvée (fig. 16). À leur place, on avait employé des colonnes corinthiennes de stuc marbré monumentales, qui rappelaient le projet le plus fastueux de la chambre à coucher d'Herrenchiemsee, avec ses colonnes, non réalisées, pour marquer l'entrée de l'alcôve. 
Figure 16

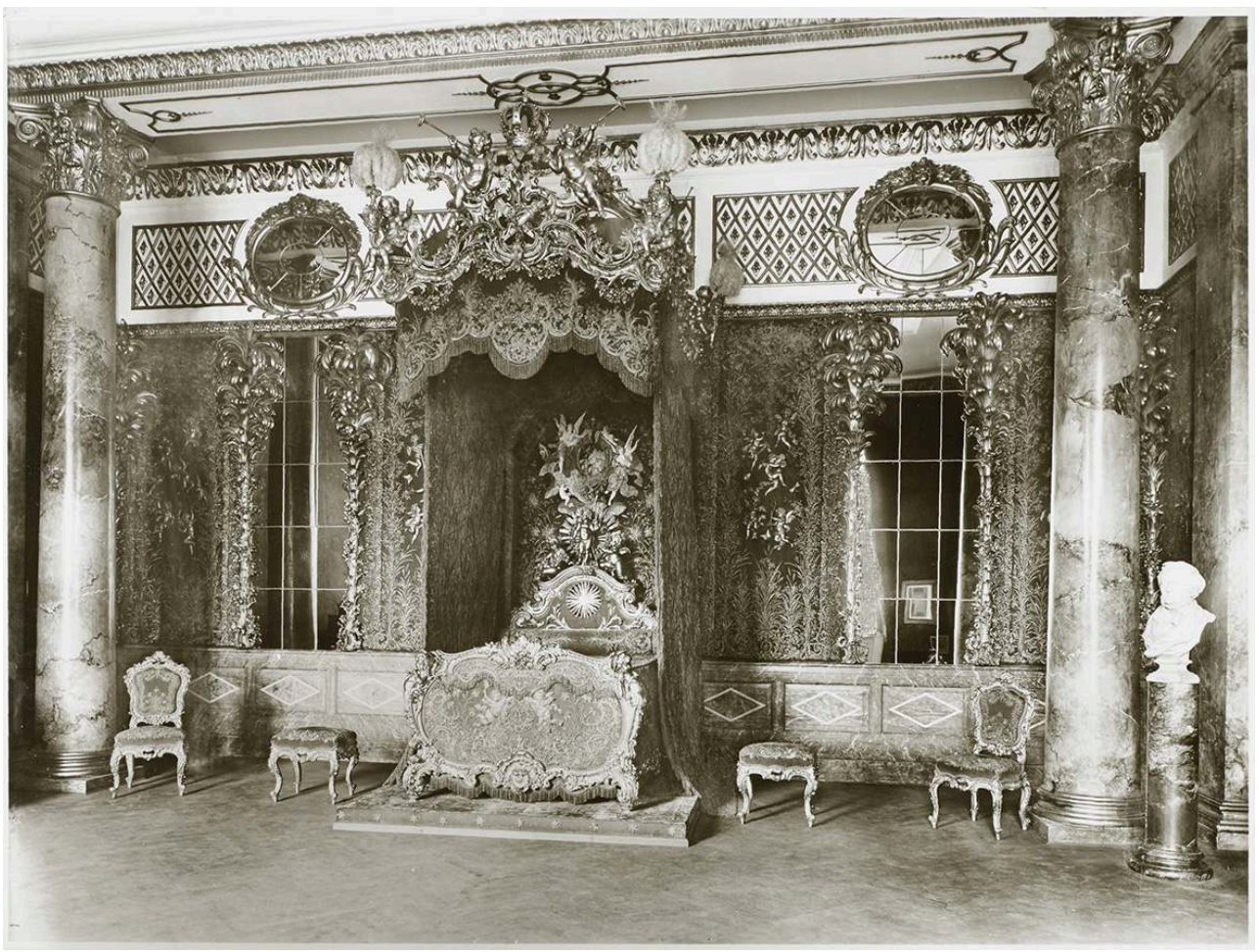

Musée national bavarois, salle Louis II (salle 48), en 1908 ou 1909.

Phot. Bayerisches Nationalmuseum. (c) Bayerisches Nationalmuseum.

En 1908, le Musée réussit à faire une acquisition pour compléter la chambre à coucher : 25 putti de bronze doré au feu, les sept papillons et les cinq libellules qui allaient avec, qui avaient également été achetés par le musée des Arts décoratifs de Strasbourg ${ }^{62}$. Après la Deuxième Guerre mondiale, l'ordre chronologique du parcours de visite, avec des salles consacrées à chaque souverain, fut abandonné (elle a été partiellement reprise en 2015). Ce n'est qu'en 1968, avec l'exposition « Le roi Louis II et l'art "63, à la Résidence de Munich, que l'on s'intéressa de nouveau à la première chambre à coucher de Linderhof. À cette occasion, le lit, dont le cadre avait subi des dommages considérables et dont des éléments de sculptures s'étaient détachés, fut prêté à l'Administration des châteaux de Bavière (die Bayerische Schlösserverwaltung) et restauré. Depuis le nouvel aménagement du musée du roi Louis II à Herrenchiemsee, en 1986, il y est exposé dans un état comparable à son état d'origine pour ce qui est de l'arrondissement de l'alcôve. Les proportions de la pièce sont ainsi correctement indiquées et l'absence de corniche et de plafond stuqué permet d'en suggérer le caractère fragmentaire (fig. 17, fig. 18). 
Figure 17

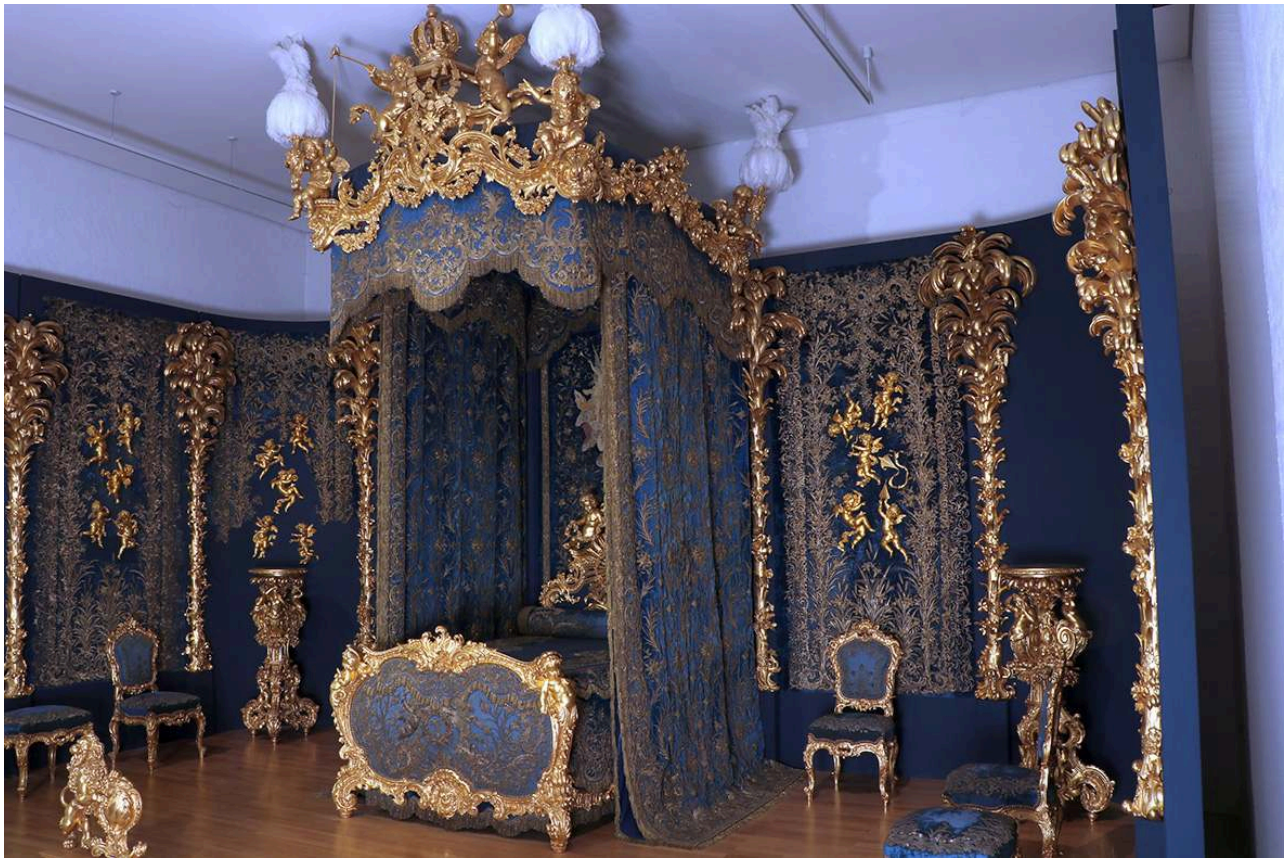

Présentation de l'ameublement conservé de la chambre à coucher, musée Louis II, vue générale, état en 2018.

Phot. Sybe Wartena. (c) Bayerisches Nationalmuseum.

Figure 18

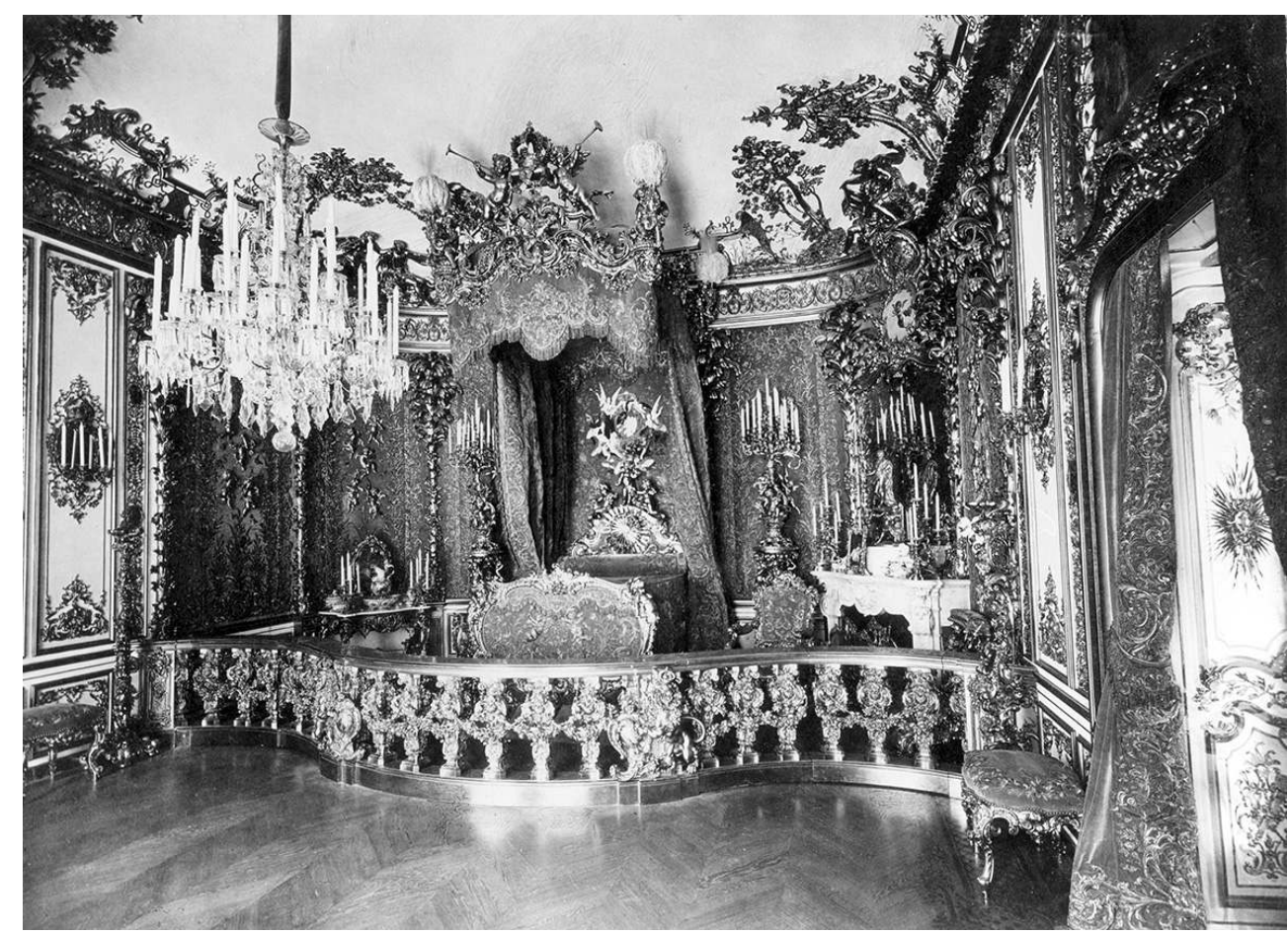

Première chambre à coucher de Linderhof, photographie de Joseph Albert, avant 1878.

(c) Bayerische Schlösserverwaltung. www.schloesser.bayern.de. 


\section{Conclusion}

Le lit de la première chambre de Linderhof est dû à l'obsession et à l'esprit tourmenté du roi Louis II, pour qui le processus de création de ses décors d'ameublement comptait plus que la possession et l'utilisation des œuvres. Jamais satisfait de ce qu'il avait obtenu, il semble que le roi était disposé à sacrifier le lit somptueux pour en donner une version encore plus grandiose, peu différente des stades antérieurs de ses chambres et gardant des aspects fondamentaux du décor et de l'iconographie. Ce n'est que grâce à des heureux hasards que cet exemple de l'éclecticisme de Louis II a été conservé.

\section{NOTES}

1. - Munich, Musée national bavarois (Bayerisches Nationalmuseum), $n^{\circ}$ d'inv. $T 2468$; et aussi de nombreux éléments s'y rapportant, revêtements muraux, sièges, chacun avec son propre numéro d'inventaire.

2. - BAUMGARTNER, Georg. Königliche Träume. Ludwig II. und seine Bauten. Munich: Heinrich Hugendubel, 1981, p. 186 ; PETZET, Michael. Gebaute Träume. Die Schlösser Ludwigs II. von Bayern. Munich : Hirmer, 1995, p. 136.

3. - La thèse selon laquelle Linderhof, sur le modèle de Versailles, aurait été conçu comme un Trianon, un lieu de retraite par rapport à Tmeicos Ettal, semble en contradiction avec cette phase $\mathrm{du}$ projet (BACHMAYER, Monika. Schloss Linderhof. Architektur, Interieur und Ambiente einer "Königlichen Villa». Thèse de doctorat. Munich : 1977, p. 59-60). Il s'agirait plutôt d'un plan de secours en cas d'échec du projet principal. Il y a toutefois des liens entre les deux projets, pour les chambres à coucher.

4. - Les références à la Résidence de Munich ne modifient que partiellement l'affirmation selon laquelle Linderhof (comme Tmeicos Ettal resp. Herrenchiemsee) aurait dû servir de cadre à une illustration idéale de la monarchie absolue française puisque l'aménagement de Linderhof dans son ensemble lui est consacré.

5. - SAILER, Ludwig. Die Bau- und Kunst-Denkmäler weiland Sr. Majestät König Ludwig II. von Bayern: ein Begleiter und andenken für den Besucher der königl. Schlösser Chiemsee, Linderhof, Neuschwanstein, Hohenschwangau und Berg. Munich : Frisch, 1886, p. 46.

6. - BAUMGARTNER, Georg. Op. cit., p. 49.

7. - Ibid., p. 43, fig. 40 ; éléments du lit exposés au musée Louis II, château d'Herrenchiemsee (HOJER, Gerhard, SCHMID, Elmar D. (éd.). König Ludwig II.-Museum Herrenchiemsee. Catalogue. Munich : Hirmer, 1986, p. 165.

8. - VATOUT, Jean. Le Palais de Versailles. Paris : Firmin Didot, 1837.

9. - WARTENA, Sybe. «Souvenirs historiques des Résidences royales de France, Bd. 1 : Palais de Versailles/«Chambre à coucher de Louis XIV »/Die Chambre de Parade in Versailles». Dans WOLF, Peter. Götterdämmerung: König Ludwig II und seine Zeit. Cat. exp., Herrenchiemsee, 14 mai-16 oct. 2011. Augsbourg : Bayerisches Staatsminisetrium für Wissenschaft, Forschung und Kunst, 2011, p. 120-121, 139-140 ( $\mathrm{n}^{\circ}$ de cat. 3.25a, 3.25c, 3.38a).

10. - N.D.T. Cité par VATOUT, Jean. Op.cit., p. 79. Il existe plusieurs manuscrits $d u x{ }^{e}$ e siècle intitulés « Description de l'ameublement du roy pour son grand appartement de Versailles, fait 
par Simon Delobel, tapissier et valet de chambre de Sa Majesté et garde des meubles de la reine », " par de Soucy », BnF, bibliothèque de l'Arsenal, Ms-5127, réserve, et BnF, Français 2291 et 2292, un autre au château de Versailles (anc. coll. Grosseuvre).

11. - «Versailles - Herrenwörth. Bettbeschreibung», Wittelsbacher Ausgleichsfonds, manuscrit 45.

12. - WARTENA, Sybe. Entwurf für das Paradebett des Prunkschlafzimmers - Vorderansicht (Projet pour le lit de parade de la chambre d'apparat, vue générale). Dans WOLF, Peter. Götterdämmerung...Op. cit., p. 139-140 (cat. 3.38b).

13. - Illustrations : vue latérale dans BAUMGARTNER, G. Op. cit., p. 136, ill. p. 237 ; Vue de devant dans WOLF, P. Götterdämmerung...Op. cit., p. 139 (cat. 3.38b). Le premier projet, à quatre montants, est inédit.

14. - Pour les tissus, un grand nombre de projets plus élaborés ont été conservés, qui ne seront pas abordés ici.

15. - BAUMGARTNER, G. Op. cit., p. 153, fig. 266 ; PETZET, M. Op. cit., p. 235.

16. - N.D.T. Franz von Seitz, 1817-1883, peintre, professeur à l'Académie des beaux-arts de Munich, créateur de costumes de théâtre, est l'auteur de décors intérieurs pour Linderhof.

17. - Bayerisches Hauptstaatsarchiv (Archives nationales de Bavière, ci-après BHStA), Geheimes Hausarchiv (GHA), Hofsekretariat 385. Compte principal de la caisse du Cabinet de S.M. le roi Louis II pour l'année (ci-après : Haupt-Rechnung) 1871. Compte supplémentaire de la caisse du Cabinet $\mathrm{du}$ roi concernant les dépenses du domaine royal de «Linderhof» (ci-après Nebenrechnung Linderhof) pour 1871, p. 26, pièce justificative $n^{\circ} 102$.

18. - Dans les comptes, il est toujours question de «Bettdeckel»; selon LANGER, Brigitte. Die Möbel der Schlösser Nymphenburg und Schleißheim. Munich : Prestel, 2000, p. 98, on devrait utiliser ici un terme du XVIII ${ }^{\mathrm{e}}$ siècle : Couvertdecke (« courtepointe »).

19. - DE SOUCY. «Ameublement du Roi, pour son Grand Appartement de Versailles, fait par Simon Delobel, tapissier et valet de chambre de sa Majesté et garde des meubles de la reine». Dans VATOUT, Jean. Le Palais de Versailles. Op. cit., p. 78-81; voir le site : https://gallica.bnf.fr/ ark:/12148/bpt6k6438600j/f89.item.r=delobel [consulté le 20/06/2019].

20. - Bien que le projet ne montre pas la doublure des rideaux, la réalisation technique a nécessité de faire deux couches séparées. Voir les rideaux dorés de la chambre de parade à Herrenchiemsee (HEINEMANN, Katharina et WARTENA, Sybe. « Goldvorhang des Prunkbettes im Paradeschlafzimmer in Schloss Herrenchiemsee ». Dans WOLF, P. Götterdämmerung... Op. cit., cat. $\mathrm{n}^{\circ} 3.43$, p. 145-146).

21. - BHStA, op GHA, Hofsekretariat 386. Hauptrechnung 1872. Nebenrechnung Linderhof 1872, p. 28, pièce justificative $\mathrm{n}^{\circ} 168$.

22. - BHStA, GHA, Hofsekretariat 385. Hauptrechnung 1871. Nebenrechnung Linderhof 1871, p. 26, pièce justificative $\mathrm{n}^{\circ} 98$.

23. - SCHMID, Elmar D. « Schloß Linderhof, erstes Schlafzimmer. Entwurf zum Prunkbett». Dans HOJER, Gerhard (éd.). König Ludwig II.-Museum Herrenchiemsee. Op. cit., p. 354, cat. nº 248.

24. - SCHICK, Afra. Möbel für den Märchenkönig. Ludwig II. und die Münchner Hofschreinerei Anton Pössenbacher. Stuttgart: Arnold, 2003, p. 210-211 ; Saalbuch Bayerisches Nationalmuseum Saal 19-23, [Guide des salles du Musée national bavarois], vers 1881, p. 95.

25. - La forme qui apparaît dans le document de 1872 pour Tmeicos Ettal a pu être créée plus tôt, sur des dessins qui n'ont pas été conservés.

26. - « Rückwand des Schlafzimmers soll ganz mit blauem Samt überzogen werden, auch die einzelnen die Felder trennenden Streifen » (BACHMAYER, Monika. Thèse cit., p. 235, annexe $\mathrm{n}^{\circ}$ 5.6.1.2, Bayerische Schlösserverwaltung Inv.-Nr. L.II.-Mus Nr. 2000).

27. - BHStA, GHA, Hofsekretariat 386. Hauptrechnung 1872. Nebenrechnung Linderhof 1872, p. 29, pièce justificative $n^{\circ} 169$. 
28. - GOERTZ, Beate. Schloss Linderhof. Die Innenraumgestaltung der beiden Schlafzimmer. Mémoire de maîtrise. Vienne : université de Vienne, 1996, p. 67, note 226.

29. - HEINEMANN, Katharina. "Entwurf für die Chambre de Parade in Herrenchiemsee » [Projet pour la chambre de parade d'Herrenchiemsee]. Dans WOLF, P. Götterdämmerung... Op.cit., p. 140-141. La peinture à l'huile de Wilhelm Hauschild correspond vraisemblablement au paiement de 1200 florins "pour la réalisation d'une peinture à l'huile, "représentant l'intérieur d'une chambre à coucher" ("für die Herstellung eines Ölgemäldes, „das Innere eines Schlafzimmers darstellend"»), enregistré pour Linderhof en 1873 (BHStA, GHA, Hofsekretariat 387. Hauptrechnung 1873. Nebenrechnung Linderhof 1873, p. 71, pièce justificative $n^{\circ} 158$ ).

30. - SCHICK, A. Op.cit., p. 210 (d'après BHStA, GHA, Hofsekretariat 387. Hauptrechnung 1873. Nebenrechnung Linderhof 1873. Pièce justificative 244). La relation entre ces dessins et la vue générale désignée « Adolf Seder » WAF VIII B39 (fig. 5) reste obscure.

31. - Bayerische Staatsbibliothek [Bibliothèque nationale de Bavière], Rar. 2160 ; voir le site : http://daten.digitale-sammlungen.de/ db/0009/bsb00092646/images/ [consulté le 20/06/2019].

32. - Voir le site : http://daten.digitale-sammlungen.de/0009/bsb00092646/images/index.html? id=00092646\&seite=65\&fip=193.174.98.30\&nativeno=\%2F\&groesser $=200 \% 25 \quad$ [consulté le 20/06/2019].

33. - Voir le site : http://daten.digitale-sammlungen.de/0009/bsb00092646/images/index.html? id=00092646\&groesser=\&fip=193.174.98.30\&no=\&seite=39 [consulté le 20/06/2019].

34. - HOJER, Gerhard. « König Ludwig II. - ein Bauherr des Historismus ». Dans HOJER, Gerhard, SCHMID, Elmar D. (éd.). König Ludwig II.-Museum Herrenchiemsee. Op. cit., p. 11-30, p. 27 (fig. 14), p. 28. Le projet d'un lit de parade pour l'Exposition universelle de Londres, en 1851, illustré par G. Hojer anticipe les caractéristiques du lit de Linderhof et peut être considéré à juste titre comme une possible source d'inspiration concrète, d'autant plus que les publications s'y rapportant étaient connues des artisans travaillant pour Louis II.

35. - Voir les sites: http://daten.digitale-sammlungen.de/0009/bsb00092646/images/ index.html?id=00092646\&seite=15\&fip=193.174.98.30\&nativeno=\%2F\&groesser=100\%25； http:// daten.digitale-sammlungen.de/0009/bsb00092646/images/index.html?

id $=00092646 \&$ seite $=25 \&$ fip=193.174.98.30\&nativeno=\%2F\&groesser $=100 \% 25 \quad$ [consultés le 20/06/2019].

36. - Photographie des bois de lit avant dorure, voir le site: https://radspieler.com/ firmengeschichte/ [consulté le 20/06/2019]. Le fait que le côté était clos par un tissu laisse supposer que voir le lit ouvert était inconcevable.

37. - Dans les palais de Munich, à l'époque de Louis II et encore de nos jours pour certains, les lits avaient été réalisés par l'atelier de Georges Jacob, à Paris, vers 1780 (LANGER, Brigitte. Die Möbel der Residenz München I. Die französischen Möbel des 18. Jahrhunderts. Munich : Prestel, 1995, cat. $\mathrm{n}^{\circ} 47,86,87$ ), et à Munich, par l'ébéniste de la Cour Nikolaus Daniel, vers 1815 (LANGER, Brigitte, et al. Die Möbel der Residenz München III. Möbel des Empire, Biedermeier und Spätklassizismus. Munich : Prestel, 1997, cat. $n^{\circ}$ 31; Ead. Die Möbel der Schlösser Nymphenburg und Schleißheim. Munich : Prestel, 2000, cat. $\left.n^{\circ} 101\right)$.

38. - BHStA, GHA, Hofsekretariat 387. Hauptrechnung 1873. Nebenrechnung Linderhof 1873. Pièce justificative $\mathrm{n}^{\circ} 244$ (omission dans la reproduction de ce document par SCHICK, A. Op. cit., p. 210).

39. - Travail de l'orfèvre Ferdinand Harrach d'après des modèles du sculpteur Philipp Perron (SELIG, Lorenz. « König Ludwig II. von Bayern ». Weltkunst, 1986-24, p. 3895-3897).

40. - GOERTZ, Beate. Op. cit., p. 50, note 166.

41. - BHStA, GHA, Hofsekretariat 385. Hauptrechnung 1871. Nebenrechnung Linderhof 1871, p. 22, pièces justificatives 82 et 83 , paiement de cinq fois 250 florins au sculpteur Franz Walker. 
42. - EVERS, Hans Gerhard, SCHMOLL, J. A., EGGERT, Klaus. Ludwig II. von Bayern: Theaterfürst König - Bauherr. Gedanken zum Selbstverständnis. Munich: Hirmer, 1986, p. 217 (d'après BHStA, GHA, Kabinettsakten Ludwigs II., K 55, L 4, Nr. 51).

43. - SCHICK, A. Op.cit., p. 232 ; Projet dans les archives de Louis II, Archives de la Bayerische Schlösserverwaltung; HEINEMANN, Katharina. «Schlafzimmer Ludwigs II. in Herrenchiemsee: Vorentwurf zum Bett Ludwigs II ». Dans WOLF, P. Op. cit., p. 150.

44. - ERICHSEN, Johannes. "Ludwig XIV. weiht der Muttergottes seine Krone ("Le vœu de Louis XIV"). Dans WOLF, P., Götterdämmerung... Op. cit., p. 146-147.

45. - N.D.T. : Il s'agit en fait du renouvellement du vœu de Louis XIII (consécration du royaume de France à Dieu par l'intermédiaire de la Vierge, le 10 février 1638) par Louis XIV, les deux scènes ayant été représentées, entre autres par Philippe de Champaigne (MINOIS, Léo. «Le vœu de Louis XIII et la naissance de Louis XIV : observations iconographiques sur la célébration du roi très chrétien ». Les Cahiers de Framespa [En ligne], 11 | 2012, mis en ligne le 23 novembre 2012, consulté le 30 janvier 2019. URL : http://journals.openedition.org/framespa/2009 ; DOI : 10.4000/ framespa.2009).

46. - On trouve pour la première fois mention de cette commande dans un rapport au roi du secrétaire de la Cour Richard Hornig, le 4 octobre, dans lequel il explique l'impossibilité d'agrandir la chambre à coucher en ne sacrifiant qu'un seul des «cabinets blancs » («weißen Kabinette ») latéraux (BHStA GHA, Kabinettsakten König Ludwigs II., Nr. 273 (d'après GOERTZ, B. Op. cit., p. 125-126, Source $\mathrm{n}^{\circ}$ 7).

47. - N.D.T. Charles-Albert de Bavière, empereur sous le nom de Charles VII de 1742 à 1745.

48. - BHStA GHA, Kabinettsakten König Ludwigs II., Nr. 273 (d'après GOERTZ, B. Op. cit., p. 126, Source $\left.n^{\circ} 8\right)$.

49. - BHStA GHA, Kabinettsakten König Ludwigs II., Nr. 273 (d'après GOERTZ, B. Op.cit., p. 126-127, Source $\mathrm{n}^{\circ} 10$ ).

50. - « daß die Stickerei der Wände genauso wie im hiesigen Reichen Schlafzimmer zu werden hat » (BHStA GHA, Kabinettsakten König Ludwigs II., Nr. 273, d'après GOERTZ, B. Op. cit., p. 126, Source $\mathrm{n}^{\circ} 8$.

51. - Inventaire BSV.

52. - «Der Plafond des Betthimmels, ebenfalls die Taube mit dem Oehlfläschchen, Strahlen, Sterne und Wolken » (BHStA GHA, Kabinettsakten König Ludwigs II., Nr. 286. D’après GOERTZ, B. Op. cit., p. 133, Source $\left.\mathrm{n}^{\circ} 32\right)$.

53. - Exception : le projet non réalisé pour les pentes du ciel de lit d'après Eugen Drollinger (SCHICK, A. Op. cit., p. 212).

54. - BHStA GHA, Kabinettsakten König Ludwigs II., Nr. 278. (GOERTZ, B. Op. cit., p. 128, Source $\mathrm{n}^{\circ} 15$ : le secrétaire de la Cour Richard Hornig au roi Louis II, le 15 février 1885.

55. - BSV, L.II.-Mus. Nr. 268.

56. - « Allerhöchsten Befehle die Einrichtungsgegenstände des Schlafzimmers Linderhof betreffend [...] Die Decke des Bettes müsse vorne und an den Seiten herabhängen " (BHStA GHA, Kabinettsakten König Ludwigs II., Nr. 286, d'après GOERTZ, B. Op. cit., p. 133, Source $n^{\circ} 32$ ).

57. - Les projets pour les deux lits d'Herrenchiemsee comportent les mêmes incertitudes sur ce détail.

58. - Bayerisches Nationalmuseum Zugangsbuch I, 1883-1888, p. 370-371.

59. - SALVISBERG, Paul von, et al. (éd). Officieller Katalog der Deutsch-Nationalen Kunstgewerbeausstellung $z u$ München 1888. Munich: Commissions-Verlag der Academischen Monatshefte, 1888 , p. 57, illustré par la fig. 81 . Les panneaux muraux de la nouvelle chambre à coucher étaient exposés séparés du lit, associés aux palmiers sculptés de l'ancienne : sur la photo, on ne voit pas bien si le revêtement mural autour du lit est identique à celui de la première chambre à coucher. 
60. - Correspondance avec l'Association bavaroise des arts décoratifs (Bayerische Kunstgewerbeverein), Note du 18 janvier 1983 dans les documents d'acquisition du Musée national bavarois avant 1945, Dok. 1743, König Ludwig II.-Ensemble (Bett). Le reportage dans le bulletin de l'Association bavaroise des arts décoratifs (Zeitschrift des bayerischen Kunst-GewerbeVereins), 1893, n 2, p. 13, mentionne seulement, pour le pavillon construit par Gabriel von Seidl : «Les tentures murales de velours bleu rebrodé d'or (à l'origine au château de Linderhof) ont été prêtées par le Musée national par l'intermédiaire du ministère royal, le mobilier et autre ameublement de chambre provenant du château d'Herrenchiemsee ont été mis à disposition par l'administration des biens royaux.» («Die ... goldgestickten Wandbehänge aus blauem Sammt (ursprünglich in Schloss Linderhof) wurden durch Vermittlung des kgl. Ministeriums vom Nationalmuseum hergeliehen; das Mobiliar und die sonstige Zimmerausstattung aus dem Schloss Herrenchiemsee wurde von der kgl. Vermögensverwaltung zur Verfügung gestellt. ») Sur l'illustration (ibid., pl. 8), on reconnaît, avec un fauteuil et un tabouret de la chambre de parade d'Herrenchiemsee, une des chaises et les palmiers de Linderhof. Le nombre de 23 objets prêtés par le Musée indique que le lit avait dû être exposé à Chicago.

61. - «Sechs geschnitzte und vergoldete Palmen aus dem alten Schlafzimmer des Schlosses Linderhof aus der Vermögensadministration des Königs Otto " (Bayerisches Nationalmuseum Zugangsbuch II, 1889-1896, p. 302).

62. - Bayerisches Nationalmuseum Zugangsbuch V, 1908-1912, p. 61 ; Correspondance dans les documents d'acquisition du BNM avant 1945, Dok. 1743, König Ludwig II.-Ensemble (Bett). Le prix d'achat était de 1250 Mark.

63. - PETZET, Michael. König Ludwig II. und die Kunst. Cat. exp., Munich, palais de la Résidence, 20 juin-15 octobre 1968. Munich : Prestel, 1968.

\section{RÉSUMÉS}

Parmi les projets de construction et d'aménagement historicistes de Louis II de Bavière, son appartement dans la Résidence de Munich et les palais de Linderhof et de Herrenchiemsee représentent respectivement le baroque français et le style rocaille (rococo). Cinq chambres ont été créées, dont la conception se nourrit de l'engouement de Louis II pour la chambre d'apparat de Louis XIV à Versailles et sa description littéraire. La plus fastueuse de ces chambres est la chambre de parade de Herrenchiemsee, avec le lit correspondant (avant-projets dès 1869, début de réalisation dès 1876 , achèvement en 1883). Le lit de la première chambre de Linderhof, conçu dès 1871 , réalisé en 1873, abandonné et remplacé dès 1884 puis donné au Musée national de Bavière en 1887, présente, en ce qui concerne la genèse de sa conception, quelques points communs avec le lit de Herrenchiemsee, bien que tous deux se réfèrent à des époques stylistiques différentes : celui de Herrenchiemsee fait écho à l'époque de Louis XIV par ses ornements, tandis que celui de Linderhof adopte le rococo. Les traits fondamentaux du programme iconographique montrent un parallèle supplémentaire entre les deux lits. On peut déceler dans les formes du lit de Linderhof une source importante, l'œuvre de Juste-Aurèle Meissonnier (1695-1750), qui fut souvent mis à profit par Louis II. Des détails importants - le matelas en crin de cheval à ressorts et la courtepointe fixée sur un cadre très élevé - en font une œuvre caractéristique du XIX ${ }^{\mathrm{e}}$ siècle.

Amongst the historicist architectural and decorative projects of King Ludwig II of Bavaria, the apartments in his Munich residence and the palaces of Linderhof and Herrenchiemsee are 
influenced, respectively, by French-style baroque and then by rococo. In these palaces, five bedrooms were created in all, largely inspired by Ludwig's admiration for Louis XIV's state bedroom at Versailles and the literary descriptions of its bed. The largest and most sumptuous of these bedrooms is the state bedroom of the Herrenchiemsee palace, with its bed. This room was projected and designed from 1869 and executed between 1876 and 1883. The bed of the first bedroom at the Linderhof palace was planned from 1871 and completed in 1873. It was then abandoned and replaced in 1884 and the first bed was given to the Bavarian National Museum in 1887. In its original design, this bed shows several similarities with the one at the Herrenchiemsee palace, although the two beds have different stylistic references: the Herrenchiemsee one is influenced by the ornamental forms of the age of Louis XIV, whilst the Linderhof bed is of rococo inspiration. Another similarity between the two beds is to be seen in the main figures in their iconographic programmes. An important source for the ornamental details of the Linderhof bed can be identified: the works of Juste-Aurèle Meissonnier (1695-1750), which often inspired Ludwig II. Significant details such as the spring and horsehair mattress or the counterpane on a frame suspended high above the bed characterize it as work of the nineteenth century.

\section{INDEX}

Mots-clés : néo-rococo, néo-baroque, lit Louis II de Bavière, lit Louis XIV, chambre de parade, colombe de la Sainte Ampoule, Linderhof, meuble sculpté, Anton Pössenbacher

Keywords : neo-Rococo, neo-baroque, bed, Ludwig II of Bavaria, Louis XIV, state bedroom, dove of the holy ampulla, Linderhof, sculpted furniture, Anton Pössenbacher

\section{AUTEUR}

\section{SYBE WARTENA}

Conservateur des meubles, instruments de musique, jeux et plans reliefs, Bayerisches Nationalmuseum Sybe.Wartena@bnm.mwn.de 